Elsevier Editorial System(tm) for Experimental Neurology

Manuscript Draft

Manuscript Number:

Title: Cerebral Vascular Injury in Traumatic

Brain Injury Article Type: SI: Traumatic Brain

Injury

Keywords: traumatic cerebral vascular injury (TCVI); traumatic brain injury (TBI), neurovascular unit (NVU); cerebrovascular reactivity (CVRx); cerebral microvasculature; microvascular injury; cerebral blood flow (CBF); controlled cortical impact (CCI); fluid percussion injury (FPI); chronic traumatic encephalopathy (CTE); hypercapnia; single photon emission computed tomography (SPECT); MRI-arterial spin labeling (MRI-ASL); susceptibility-weighted imaging (SWI); mild TBI (mTBI); transcranial Doppler (TCD); functional Near InfraRed Spectroscopy (fNIRS); hypercapnia

Corresponding Author: Ramon Diaz-Arrastia, MD, PhD

Corresponding Author's Institution: Uniformed Services University of the Health

Sciences First Author: Kimbra Kenney, MD

Order of Authors: Kimbra Kenney, MD; Franck Amyot, PhD; Margalit Haber, PhD; Angela Pronger, BA; Tanya Bogoslovsky, MD, PhD, Carol Moore, MA; Ramon Diaz-Arrastia, MD, $\mathrm{PhD}$

Abstract: Traumatic cerebral vascular injury (TCVI) is a very frequent, if not universal, feature after traumatic brain injury (TBI). It is likely responsible, at least in part, for functional deficits and TBI-related chronic disability. Because there are multiple pharmacologic and nonpharmacologic therapies that promote vascular health, TCVI is an attractive target for therapeutic intervention after TBI. The cerebral microvasculature is a component of the neurovascular unit (NVU) coupling neuronal metabolism with local cerebral blood flow. The NVU participates in the pathogenesis of TBI, either directly from physical trauma or as part of the cascade of secondary injury that occurs after TBI. Pathologically, there is extensive microvascular injury in humans and experimental animal, identified with either conventional light microscopy or ultrastructural examination. It is seen in acute and chronic TBI and even described in chronic traumatic encephalopathy (CTE). Non-invasive, physiologic measures of cerebral microvascular function show dysfunction after TBI in humans and experimental animal models of TBI. These include imaging sequences (MRI-ASL), Transcranial Doppler (TCD), and Near InfraRed Spectroscopy (NIRS), etc. Understanding the pathophysiology of TCVI, a relatively under-studied component of TBI, has promise for the development of novel therapies for TBI. 


\title{
Cerebral Vascular Injury in Traumatic Brain Injury
}

\author{
Kimbra Kenney, MD; Franck Amyot, PhD; Margalit Haber, \\ $\mathrm{PhD}$; Angela Pronger, BA; Tanya Bogoslovsky, MD, PhD, \\ Carol Moore, MA; Ramon Diaz-Arrastia, MD, PhD
}

Center for Neuroscience and Regenerative Medicine

Department of Neurology

Uniformed Services University of the Health Sciences

4301 Jones Bridge Road

Bethesda, MD 20814

Corresponding author:

Ramon Diaz-Arrastia, M.D., Ph.D.

Dept. of Neurology, USUHS

4301 Jones Bridge Road

Bethesda, MD 20814

Tel: $\quad 301-295-55537$

Email: ramon.diaz-arrastia@usuhs.edu 


\section{ABSTRACT:}

Traumatic cerebral vascular injury (TCVI) is a very frequent, if not universal, feature after traumatic brain injury (TBI). It is likely responsible, at least in part, for functional deficits and TBI-related chronic disability. Because there are multiple pharmacologic and nonpharmacologic therapies that promote vascular health, TCVI is an attractive target for therapeutic intervention after TBI. The cerebral microvasculature is a component of the neurovascular unit (NVU) coupling neuronal metabolism with local cerebral blood flow. The NVU participates in the pathogenesis of TBI, either directly from physical trauma or as part of the cascade of secondary injury that occurs after TBI. Pathologically, there is extensive cerebral microvascular injury in humans and experimental animal, identified with either conventional light microscopy or ultrastructural examination. It is seen in acute and chronic TBI, and even described in chronic traumatic encephalopathy (CTE). Non-invasive, physiologic measures of cerebral microvascular function show dysfunction after TBI in humans and experimental animal models of TBI. These include imaging sequences (MRI-ASL), Transcranial Doppler (TCD), and Near InfraRed Spectroscopy (NIRS), etc. Understanding the pathophysiology of TCVI, a relatively understudied component of TBI, has promise for the development of novel therapies for TBI.

Key Words (minimum 10): traumatic cerebral vascular injury (TCVI), traumatic brain injury (TBI), neurovascular unit (NVU), cerebrovascular reactivity (CVRx), cerebral microvasculature, microvascular injury, cerebral blood flow (CBF), controlled cortical impact (CCI), fluid percussion injury (FPI), chronic traumatic encephalopathy (CTE), hypercapnia, single photon emission computed tomography (SPECT), MRI-arterial spin labeling (MRI-ASL), susceptibilityweighted imaging (SWI), mild TBI (mTBI), transcranial Doppler (TCD), functional Near InfraRed Spectroscopy (fNIRS), hypercapnia 


\section{INTRODUCTION:}

TBI results in an enormous burden on patients, their families, and society. The magnitude of this problem has led to extensive preclinical research and numerous clinical trials aimed at developing therapies to improve functional outcome after TBI.(Narayan et al, 2002). In animal models, therapeutic interventions aimed at modulating molecular pathways identified to be induced after TBI have been successful in limiting the extent of injury and improving neurologic recovery.(Marklund et al, 2006;McIntosh, 1993;McIntosh et al, 1998) These experimental observations constitute a convincing demonstration of the principle that not all neural damage occurs at the time of injury, and that opportunity exists for therapeutic intervention. Unfortunately, Phase III clinical trials of these therapies in patients with severe brain injuries have failed to demonstrate efficacy.(Doppenberg and Bullock, 1997) The failure of these clinical trials have led to several NINDS and DoD consensus conferences over the past 15 years.(DiazArrastia et al, 2013;Hyder et al, 2007;Narayan et al., 2002;Saatman et al, 2008) These conferences have concluded that (1) validated imaging and biochemical biomarkers will be critical for the development of effective therapies; and (2) therapies should be targeted at specific injury mechanisms, since it is unlikely that a single therapy will be effective in such a heterogeneous condition.(Diaz-Arrastia et al., 2013;Hyder et al., 2007) Biomarker-driven therapeutic approaches remain critically necessary in order to: 1) prove that the therapy is actually engaging its proposed molecular target; and, 2) provide proof of principle by demonstrating efficacy on a pharmacodynamic biomarker. Since a biomarker is mechanistically closer to the biological effect of the therapy than clinically relevant outcome measures, early phase studies can achieve adequate power with modest sample sizes. Relevant biomarkers can be 
useful for fine-tuning important issues such as dose, timing, and duration of therapy before launching large and expensive Phase III trials.

While the complex molecular and cellular mechanisms responsible for functional deficits after TBI are not fully understood, substantial data point to traumatic cerebral vascular injury (TCVI) underlying a significant fraction of TBI-related disability. TCVI is a well-established but relatively understudied endophenotype of TBI. The cerebral vasculature is a highly plastic tissue making TCVI an attractive target for therapeutic intervention after TBI. There are multiple well established pharmacologic and non-pharmacologic therapies which ameliorate blood vessel injury and promote vascular health. These therapies may prove beneficial to TBI patients. Candidate pharmacologic therapies targeted at improving cerebrovascular function include PDE5 inhibitors, HMG-CoA reductase inhibitors, HDL mimetics, and PPAR- $\gamma$ agonists, among others.(Nicolakakis et al, 2011;Nicolakakis and Hamel, 2011) Non-pharmacologic therapies targeted at improving vascular function are also attractive therapeutic interventions and include aerobic exercise, dietary interventions, and nutraceuticals such as omega-3 fatty acids.(Wang et al, 2014). This review will focus on preclinical and clinical data examining microvascular injury after TBI as well as survey candidate therapies for acute and chronic TCVI.

\section{Theoretical framework, the neurovascular unit}

Studies of the physiologic relationships among neuronal networks, blood vessels, and glia date to the late $19^{\text {th }}$ and early $20^{\text {th }}$ centuries.(Hawkins and Davis, 2005; Roy and Sherrington, 1890; Villringer and Dirnagl, 1995) The micro-network that regulates blood flow, vascular 
permeability and angiogenesis in the central nervous system (CNS) has been coined the neurovascular unit (NVU).(Cohen et al, 1996;Hamel, 2006;Lecrux and Hamel, 2011) The brain is critically dependent on a steady blood supply and, unlike other organs, is intolerant to fluctuations in blood flow. In order to maintain physiologically adequate cerebral perfusion, multiple sophisticated homeostatic mechanisms have evolved. The connection between cerebral blood flow (CBF) and CNS metabolism was postulated by Roy and Sherrington at the end of the $19^{\text {th }}$ century(Roy et al., 1890) and confirmed by Olesen et al in the 1970s(Olesen et al, 1971) and Sokoloff in the early 1980s.(Sokoloff, 1981) Since then it has been well established that CBF is tightly coupled to increased neuronal activity.(Tan et al, 2014) A constant cerebral blood flow is maintained despite wide changes in perfusion pressure.(Lassen, 1959;Tan et al., 2014) This system, as originally described, is composed of the cerebral blood vessel wall, circulating blood elements, glia and neurons, but has now been expanded to include additional cell types, including pericytes, microglia and perivascular astrocytes. Additionally, it includes specialized cellular compartments such as the endothelial glycocalyx, the endothelial lining of cerebral capillaries, capillary tight junctions, and the capillary basement membrane, all of which contribute to the integrated system which functions in support of each other.(Golding, 2002) Morphologically, cerebral arterioles and capillaries have 3 layers: tunica intima, tunica media and tunica adventitia. The tunica intima, the innermost layer adjacent to the vessel lumen, is made up of a single layer of endothelial cells surrounded by a basement membrane composed of extracellular matrix proteins (e.g. collagen, laminin and heparin sulphate proteoglycans). The tunica media, or muscular component, is made up of elastic laminae and smooth muscle cells. Penetrating arterioles have one or two smooth muscle cells per circumference. In capillaries, there are no smooth muscle cells, as pericytes take their place. The tunica adventitia is the 
outermost layer and includes terminal nerve fibers and surrounding connective tissue.(Golding, 2002) Endothelial cells respond to hemodynamic forces (e.g. vasoactive stimuli such as norepinephrine and prostaglandins or physical forces such as stretch and pressure) via the release of factors that promote constriction or dilation. One of the primary vascular dilation factors that endothelial cells release is nitric oxide (NO), which is produced by endothelial NO synthase (eNOS).(Dimmeler et al, 1997) The cerebral vasculature is innervated by neurons whose terminals release neurotransmitters (e.g. norepinephrine and serotonin for vasoconstriction; and acetylcholine, substance $\mathrm{P}$, vasoactive intestinal polypeptide for vasodilation) that diffuse into the tunica media and act on receptors in the smooth muscle cell layer to elicit either vasoconstriction or dilation. Based on local activity and needs, basal forebrain neurons release vasoactive mediators on cortical microvessels and supporting astroctyes to modulate microvascular tone.(Stanimirovic and Friedman, 2012)Thus neuronal metabolism and activity are tightly coupled to local cerebral blood flow.(Villringer et al., 1995)

The NVU participates actively in the pathogenesis of many brain disorders. It is hypothesized that vascular events may be a primary cause of neurological diseases whose principal pathology was previously thought to be primarily neuronal.(Xing et al, 2012) Common human diseases such as hypertension and diabetes damage the cerebral microvasculature and prominently influence the expression of neurodegenerative disorders (e.g. Alzheimer's disease).(Breteler, 2000;Zlokovic, 2008) The NVU has been an intense focus of research in multiple acute and chronic neurologic disorders, including stroke, dementia, epilepsy, Parkinson's disease, amyotrophic lateral sclerosis and demyelinating disease.(Xing et al., 2012) While difficult to distinguish cause and effect, several clinical studies suggest that dysfunction of 
the NVU is a cause, rather than simply a consequence, of neurodegeneration.(del Zoppo, 2013;Lecrux et al., 2011)

Vascular dysfunction can occur as a result of primary neuronal dysfunction or may precede the neuronal dysfunction. Disruption of the NVU in a genetic disorder, cerebral autosomal dominant arteriopathy with subcortical infarcts and leukoencephalopathy (CADASIL), which produces primary microvasculopathy provides direct evidence that vascular dysfunction alone can cause neurodegeneration.(Stanimirovic et al., 2012) CADASIL is a hereditary disease of small cerebral vessels that is associated with migraine, strokes, dementia and mood disorders. It is the most common form of heritable stroke and vascular dementia.(Marchesi, 2014) With an onset generally in middle age (40s to 60s), CADASIL is characterized by a progressive dementia, clinically very similar to Alzheimer's disease, with death generally 15-20 years after disease onset. Neuroimaging shows leukoaraiosis in subcortical white matter, before clinical symptoms develop. Ultrastructurally, the primary pathology is thickening of small penetrating cerebral and leptomeningeal arteries as well as granular osmophilic accumulation in the basement membrane and arterial smooth muscle cells of those arteries. The accumulated material includes polypeptide segments of the extracellular domain of the NOTCH 3 protein. CADASIL is associated with germline mutations in the NOTCH 3 gene, a transmembrane receptor primarily expressed in cerebral arterial smooth muscle cells and pericytes.(Chabriat et al, 2009) It is thought that the mutant NOTCH 3 protein is toxic to smooth muscle cells of the small cerebral arterioles. The resultant microvascular abnormalities cause progressive microscopic ischemia of the areas supplied by the abnormal vessels. This results in neuronal death and neurodegeneration.(Marchesi, 2014) 
Pathophysiology of NVU injury after TBI

The pathophysiology of TBI has been divided into primary and secondary injury. Primary injury results from the physical impact causing direct biomechanical injury to cerebral tissues (neurons, glia and vessels). Secondary injury results from the molecular and cellular responses to the initial injury, resulting in a physiologic cascade that furthers neural injury(Besancon et al, 2008;Ellison et al, 1989;Golding et al, 1999;Inoue et al, 1991;Pappius et al, 1988) TCVI can result from both primary (blunt force) and secondary injury (e.g. blood brain barrier disruption, increased intracellular calcium, mitochondrial dysfunction, neuroinflammation).(Golding, 2002) Ultrastructurally, the blood brain barrier (BBB) is made up of the endothelial tight junction complex which seals the intracellular space between endothelial cells and regulates transcellular transport. Other NVU cells, including astrocytes, pericytes, microglia and neurons, also regulate the BBB through multiple different signalling cascades. After TBI, the changes seen in the BBB have a biphasic mode of action. Immediate alterations are caused by direct physical damage to endothelial cells followed later by changes from secondary injuries in other elements of the NVU (neurons, astrocytes, perictyes, microglia and the extracellular matrix).(Shlosberg et al, 2010) Diminished CBF and focal tissue hypoxia is a common precipitant of NVU pathophysiology and is mediated through multiple pathophysiologic cascades (e.g. BBB disruption, edema, focal ischemia).(Bartnik-Olson et al, 2014;Lecrux et al., 2011;Xing et al., 2012) In response to injury, the NVU induces transcription and inflammatory factors that promote local angiogenesis in addition to immediate vascular reactions that increase local blood flow and oxygen supply.(Chodobski et al, 2011;Giza and 
Hovda, 2014;Lenzlinger et al, 2002;Rodriguez-Baeza et al, 2003;Shlosberg et al., 2010)

However, the NVU also attempts to repair itself and restore homeostasis through mechanisms with potentially unintended deleterious consequences, resulting in increased BBB disruption, cerebral water and ion redistribution, and oxidative stress. These alterations can collectively enhance secondary injury of the NVU if homeostasis is not quickly restored. Ultimately, impaired or unchecked signalling cascades between neurovascular and glial elements results in further local injury.(Shlosberg et al., 2010) Indirect evidence supports the notion that persistent post-concussive symptoms after mild TBI (mTBI) in the pediatric population is at least partially caused by ongoing alterations in the NVU; these include reduced cerebral blood flow and cerebral blood volume, and impaired axonal/neuronal metabolism and perfusion.(Bartnik-Olson et al., 2014) Mechanisms underlying the reduced blood flow and volume are not completely understood. Potential etiologies include altered cerebrovascular autoregulation with or without associated vasospasm or regional perfusion imbalances. These changes are oftentimes detected remote from the point of impact and likely represent secondary changes in the NVU after TBI.

\section{Pathology of microvascular injury in acute TBI—primary injury}

Preclinical studies of acute changes. Microvascular injury is a near universal feature of preclinical TBI, and has been reported in nearly all animal models of TBI to date including impact acceleration,(Baranova et al, 2008;Gao et al, 2010) fluid percussion injury (FPI),(Park et al, 2009; Wei et al, 2009) and controlled cortical impact (CCI)/(Cherian et al, 2011;Schwarzmaier et al, 2010) models in rodents; FPI in cats(Wei et al, 1980) as well as lateral head acceleration in baboons.(Maxwell et al, 1988) The FPI model in rats shows a diffuse decrease in cerebral microvascular density in the ipsilateral hemisphere with preserved density in the contralateral 
hemisphere.(Park et al., 2009) Two FPI intensities were assessed (2 and 3 atm) at 24 hours and 2 weeks after injury (Fig. 1). Both injuries show similar loss in microvascular density (16.9\% \pm $3.8 \%$ at $2 \mathrm{ATM}$ and $15.7 \pm 1.3 \%$ at $3 \mathrm{ATM}$ ) compared to sham injured animals.

Immunohistochemical analysis reveals a reduction in capillary number/density up to $4 \mathrm{~mm}$ distal from the point of impact. These cerebral microcirculatory deficits persist for three weeks in the animals injured at higher pressure ( 3 atm). The animals injured with a 2 atm force show improved or reversed cerebral microcirculatory deficits by three weeks.(Park et al., 2009)

Structural changes to the cerebral microvasculature seen in preclinical models include vacuolation, discontinuity, degeneration or regeneration, and pericytes with necrosis and migration. Adjacent vascular smooth muscle cells remain largely unchanged.(Dewitt and Prough, 2003) Early studies with FPI in Sprague-Dawley rats show petechial hemorrhages associated with small venules, dark shrunken neurons, and swollen astrocytes in areas surrounding the contusion. Ultrastructural analysis reveals evidence of mechanical damage to the vessel walls early after injury in areas with irreversible neuronal injury.(Dietrich et al, 1994) In rodents, microthrombi are seen in $70 \%$ of venules and $30 \%$ of arterioles.(Schwarzmaier et al., 2010) In a separate study of FPI in rats, microvascular density is markedly reduced (mean $57 \%$ loss) within a cortical contusion and associated with a robust inflammatory response. The reduction is interpreted as focal microvascular destruction in the contused tissue.(Corsellis and Brierley, 1959) Ultrastructural microvascular changes reported in primates include endothelial disruption and swelling of perivascular astrocytes in areas with petechial hemorrhages. Electron microscopy (EM) changes in endothelial cells are seen as early as 3 hours after injury but persist for 1 week after injury.3187\} In rats, approximately $40 \%$ of pericytes migrate from 
microvascular locations to thinning areas of the basal lamina within hours after controlled cortical impact injury.(Dore-Duffy et al, 2000) FPI in cats causes crater-like defects in segments of pial arteries and arterioles. Abnormal endothelial cells have large cytoplasmic vacuoles as well as pinocytotic vesicles.(Wei et al., 1980) Using a corrosion casting technique and scanning electron microscopy (SEM), Sangiorgi et al(Sangiorgi et al, 2013) describe microvascular injury similar to that found in human tissue by Rodriguez-Baeza et al.(Rodriguez-Baeza et al., 2003) While the human study involved convenience samples with tissue collected between 1 and 20 days after TBI, the cerebral vasculature in the rodent study was cast at 2 time points after CCI, 3 and 12 hours. At 3 hours, changes in morphology and vascular architecture are seen primarily in medium-sized and capillary vessels. Focal swelling of perforating vessels, widening of intercellular junctions and structural changes of endothelial cells are also observed. The analysis reveals marked extravasation of resin consistent with areas of subarachnoid, subdural and intraparenchymal petechiae and haemorrhage. These are thought to result from direct mechanical damage of the vessel wall from the cortical impact (Fig. 2). By 12 hours, extravasation of resin is no longer visible (Fig. 3). The predominant findings are abnormal vessels with focal constriction and distal calibre reduction in the subcortical microvasculature adjacent to the subcortical white matter. These are thought to reflect cytotoxic edema from secondary brain injury.(Sangiorgi et al., 2013)

While largely unaffected, vascular smooth muscle cells from the middle cerebral artery show occasional minor necrotic changes as well as inhibition of gap junction coupling in rat after FPI.(Golding, 2002;Yu et al, 2014) A recent study of microvascular pathology in the CCI model in adult rats studied in both acute and chronic time points shows microvascular injury associated 
with inflammation, blood-brain barrier (BBB) disruption and progressive white matter injury.(Glushakova et al, 2014) The acute injury causes focal microbleeds that gradually increase over 3 months. Immunohistochemical changes include endothelial damage, BBB damage, activation of microglia, gliosis and aggregates of activated macrophages. At 3 months after injury, the microbleeds are surrounded by glial scars, focal hemosiderin deposits and marked loss of myelin in white matter including the corpus callosum. The authors propose that delayed, focal microbleeds after TBI may result from chronic microvascular injury eventually leading to neurotoxic iron deposition and neuronal loss.(Glushakova et al., 2014)

Human neuropathological observations in acute/subacute TBI. Features of TVI observed in many experimental TBI models are similar to those seen in human specimens. While frequently underemphasized, TCVI is a near universal feature of severe human TBI (Fig. 4).(Graham et al, 2002) Pathological reports of TCVI resulting from fatal TBI are found predominantly in older literature.2539\} Pathological reports from nonfatal TBI are found less frequently. There are reports from individuals who suffered concussions and died soon thereafter from either trauma or non-trauma related complications (such as pulmonary emboli) or from cerebral tissues surgically removed during lobectomy or hematoma evacuation of non-fatal TBIs.(Oppenheimer, 1968;Stein et al, 2002) TCVI is a prominent finding in patients who die in the acute period after injury.(Oppenheimer, 1968;Rodriguez-Baeza et al., 2003;Stein et al, 2004) Oppenheimer(Oppenheimer, 1968) noted that the TBI patient brains he examined, ranging from mild to severe TBI, show microscopic perivascular petechial hemorrhages even when macroscopic hemorrhage was absent on gross examination of the brain. Small capillaries and vessels exhibit more perivascular microhemorrhages than larger vessels after TBI. In lobectomy 
specimens surgically excised between 1 hour and 9 days in patients with traumatic hematomas, there are abundant microhemorrhages evident. The microhemorrhages are not apparent grossly and contain intravascular fibrin microthrombi in small and medium-sized arterioles and venules. The highest concentration of microhemorrhages are in pericontusional tissue with moderate amounts both within the contusion itself and diffusely throughout the ipsilateral, noncontused cerebral hemisphere. Intravascular microthrombi peak at 48 hours after TBI but persist for at least 9 days.(Stein et al., 2004) Microscopic examination identifies abundant intravascular microthrombi in the arterioles and venules, primarily affecting microvessels of 6 to $100 \mu \mathrm{m}$ diameter. In a second study of samples from non-contused or hemorrhagic frontal and hippocampal sections in cases of fatal TBI with death within 48 hours of injury, intravascular microthrombi were seen in every section.(Stein et al., 2004) The microthrombi were found with varying densities, clustering adjacent to areas with pyknotic neurons. The clusters were not associated ischemic changes in nearby glia, atypical for cerebral infarction. The density of the intravascular microthrombi is highly correlated with focal areas of isolated cortical neuronal necrosis. This strong correlation suggests a possible causal link between the microthrombi and neuronal death.(Stein et al., 2004) It is thought that intravascular microthrombi result as a secondary injury, promoted by the locally injured cerebral microvasculature.(Dietrich et al., 1994)

Rodriquez-Baeza and colleagues studied the ultrastructure of cortical microvasculature among 10 fatal cases of TBI between 1 and 20 days after injury.(Rodriguez-Baeza et al., 2003) He investigated corrosion casts of the cerebral vasculature of fatal TBI and non-injured healthy controls with SEM (Fig. 5, 6). Three primary morphological changes of the cerebral microvasculature are seen in TBI brains compared to healthy controls: 1) longitudinal folds; 2) 
sunken vascular surfaces with craters; and 3) flattening and reduction of the cerebral microvascular lumen. These alterations are restricted to arterioles and capillaries of the middle and deep cortical vascular zones. No changes are observed in pial vessels or the initial course of cortical arteries in the subpial zone. The folds observed are usually parallel to the longitudinal axis of the vessel. When present, the folds are evident around the full circumference of the vessel, frequently seen on vessels with a reduced diameter. The capillary bed, including both precapillary and capillary vessels, is frequently altered and appeared flattened and tortuous with loss of branching and interconnections, and more abundant blind endings. The crater indentations are seen primarily at the junction between endothelial cells.(Golding, 2002) Endothelia from TBI patients, visualized using immunocytochemistry, are irregular without a smooth appearance. Vessels appear flattened with longitudinal folds and thinned lumen. These changes correspond to the flattened and irregular folds seen in the corrosion casts. Immunocytochemical analysis also reveals thickening of endothelial cell membranes and apoptosis of the cells similar to the changes observed with SEM of corrosion casts.(Rodriguez-Baeza et al., 2003)

Recent electron micrograph (EM) studies of humans describe the following changes in the human capillaries and perictyes after severe TBI: thickening of the basement membrane (approximately 3 to 8 times the width as seen in healthy controls); matrix disorganization with bifurcation and reduplication of the capillary basement membrane (BM); rarefaction, vacuolization and splitting of the BM; pericyte hypertrophy with increased vacuolar and vesicular transport; pericyte degeneration with discontinuous pericyte plasma membrane; rarefaction and focal necrosis of pericytes; occasional phagocytic pericytes; lipofuscin and lipid granular deposits in perictye cytoplasm.(Castejon, 2011;Castejon, 2014) Changes seen by EM in the subacute stage (7-30 days after injury) after TBI include extensive vascular changes: fibrous 
changes in the vascular wall, corrugated internal elastic lamina, apoptotic endothelial cells, condensed nuclei, degradation of the extracellular matrix, and heterophagy. These changes are thought to represent both apoptotic as well as regenerative features occurring simultaneously in the same areas 4 weeks after TBI.(Danaila et al, 2013) Collectively, these observations indicate widespread cerebral microvascular injury after TBI.(Castejon, 2011;Castejon, 2014;Danaila et al., 2013;Rodriguez-Baeza et al., 2003)

\section{Pathology of cerebral microvascular injury in chronic TBI-Cerebral microvasculature repair}

Injured blood vessels respond to TBI through local repair (Table 1). This includes vascular remodelling and angiogenesis. The local response involves increased production of extracellular matrix proteins and components of the basement membrane, including tenascin. There is accompanying increases in the vessel wall thickness and organization as well as changes in matrix composition. The cerebral microvasculature carries out this repair thorough increased protein synthesis along with cell hypertrophy and hyperplasia.(Golding, 2002) These time-dependent responses are absent when the injury is fatal within minutes but are regularly observed within 3 hours to 7 weeks after TBI. The vascular changes can be used to date the injury injury.(Hausmann and Betz, 2000) In animal models, pharmacologic therapies which protect the cerebral microvasculature or promote angiogenesis; such as erythropoietin,(Xiong et al, 2010) statins,(Wu et al, 2011) or sildenafil(Ding et al, 2008;Pifarre

et al, 2010;Zhang et al, 2002) promote neurologic recovery, even when administered too late to decrease lesion volume. 


\section{Pathology of cerebral microvascular injury in chronic traumatic encephalopathy (CTE)}

Prominent cerebral microvasculopathy is also noted in individuals diagnosed with CTE, including active duty service members after blast exposure(s) and athletes after multiple mTBIs.(Goldstein et al, 2012;McKee et al, 2013) Perivascular tau aggregations are recognized as one of the pathologic hallmarks of CTE that distinguishes it from other neurodegenerative disorders characterized by a tauopathy such as Alzheimer's disease and frontotemporal dementia. CTE was first described in retired professional boxers and initially called punch drunk syndrome(Martland, 1928) and then dementia pugilistica.(Corsellis et al., 1959;Corsellis et al, 1973) CTE is the currently accepted term as this syndrome is not unique to boxers. Vascular changes, such as thickened perforating arteries, absence of nuclei in vascular cells and diffuse hyaline vascular changes throughout cortices were noted even in the first cases reported.(Corsellis et al., 1973) A review of cerebral vasculature changes among CTE cases in the Corsellis collection show diffuse perivascular collections of hemosiderin, either within macrophages, neuroglia or extracellular space, particularly within the meninges, subpia and cerebellar folia.(Adams and Bruton, 1989) In sports-related cases of CTE and death within 6 months of mTBIs, vascular changes are seen including striking perivascular microgliosis and astrocytosis, neurofibrillary tangles (NFTs) and spindle-shaped neurites around small vessels in the depths of cortical gyri, as well as scattered hemosiderin-laden macrophages perivascularly.(McKee et al, 2014) In a case series of 4 military, blast-associated CTE cases, McKee described the same perivascular accumulations as well as perivascular lymphocytic cuffing, hemosiderin-laden macrophages within cerebral vessel walls and focal dystrophic calcification of penetrating small vessels in the thalamus and deep cortical white matter.(McKee and Robinson, 2014) A mouse model of blast exposure also demonstrates prominent cerebral 
microvascular pathology pathology.(Goldstein et al., 2012) In the blast-exposed mice, ultrastructural pathology of hippocampal capillaries show dysmorphic capillaries with thickened tortuous basal lamina, abnormal pericytes, and abnormal endothelial cells with dysmorphic nuclei. Additionally there are frequent accumulations of lipofuscin and inclusion bodies in perivascular processes as well as vacuolated astrocytic end feet processes.(Hausmann et al., 2000) To date, there have not been any reports of ultrastructural changes in CTE.

While it is impossible to infer causality from human neuropathologic studies, these studies support our hypothesis that cerebral microvascular injury is near ubiquitous after TBI in both humans and experimental animal TBI models. Further neuropathological studies focusing on the cerebral microvasculature need to be performed to definitively describe the extent and time course of TCVI.

\section{Non-invasive assessment of TCVI.}

Currently, there are two major methods to indirectly measure TCVI in humans and experimental models, functional neuroimaging and assessment of cerebrovascular reactivity (CVRx). Novel neuroimaging sequences have been coupled with dynamic procedures that

mimic or induce increased physiologic demand to visualize the response of the cerebral microvasculature; this method gives excellent spatial resolution. Measure of CVRx, by contrast, provides superior temporal resolution by measuring the ability of the cerebral microvasculature to increase blood flow to respond to signaling, chemical or otherwise, as would physiologically occur via intact cerebral microvasculature.

Neuroimaging and cerebral vascular reactivity studies in experimental animals

Neuroimaging studies have consistently shown reduced cerebral blood flow (CBF) acutely after 
experimental TBI, through multiple modalities, including functional MRI(Hayward et al, 2011;Pasco et al, 2007) and laser Doppler flowmetry.(Thomale et al, 2002) Pasco et al quantified regional hypoperfusion and reduced regional cerebral blood volume (CBV) starting at 5 hours after FPI in rats with dynamic susceptibility contrast MRI. They found that CBF and CBV normalized by day 3 after injury.(Pasco et al., 2007) Using arterial spin labeling (ASL) coupled with susceptibility weighted imaging (SWI), one can measure longitudinal changes in hemodynamics over days to weeks. Absolute $\mathrm{CBF}$, as measured via continuous ASL, varies over time in FPI rats. At 6 hours, CBF in the perilesional cortex decreases by $54 \%$ relative to sham injured controls. CBF returns to baseline by 24 hours after injury. Over the next 2 weeks, $\mathrm{CBF}$ decreases again to $75 \%$ of sham controls in the lesion. The pattern is similar in the ipsilateral hippocampus with reduction in CBF at 6 hours, increased CBF at 24 hours and then reductions in $\mathrm{CBF}$ by 48 hours that return to baseline values at 2 weeks. Contralaterally, there are similar but milder changes with initial hypoperfusion, followed by normalization at $24-48$ hours and then followed by a more extended hypoperfusion which eventually normalize by 2 weeks. Of interest, CBV does not change in FPI compared to sham-injured control animals over the same 2-week period.(Hayward et al., 2011) Immunohistochemical analysis of small capillaries in the perilesional tissue shows a marked reduction of small vessel density 6 hours after injury. This is followed by a slight increase in small vessel density at 2 weeks compared to baseline (Fig. 7).

CVRx can also be assessed in experimental models using in vivo microscopy through cranial windows. These windows allow for direct visualization of and access to the pial microvessels. In these studies, the animals are anesthetized and intubated. They are then administered hypercapnia challenge by introducing 3-5\% carbon dioxide $\left(\mathrm{CO}_{2}\right)$ admixed with 
room air. The changes in the pial microvasculature is then measured through the cranial window. One week after injury, the experimentally injured animals show a significant loss of vessel dilatation in response to hypercapnia compared to sham injured animals.(Baranova et al., 2008; Wei et al., 2009) Other studies report that TBI causes a loss of vasodilation to acetylcholine(Baranova et al., 2008;Gao et al., 2010; Wei et al., 2009)2545\} adenosine, and the direct nitric oxide (NO) donor sodium nitroprusside (SNP), all potent stimulators of vascular dilation.(Wei et al., 2009) These changes persist for up to 3 weeks after injury with most vessels showing $50 \%$ or greater reduction in their vasodilator response.(Kontos et al, 1980; Suehiro et al, 2003; Ueda et al, 2003; Wei et al., 2009)

Interestingly, cerebrovascular dysfunction correlates with behavioral deficits in vestibulomotor tasks and cognitive deficits in the Morris Water Maze.(Wei et al., 2009) In these studies, the deficit in the vasodilatory response to acetylcholine and hypercapnia induced by TBI is quantitatively greater than the deficit in vasodilatory response to SNP.(Wei et al., 2009) This suggests that injured endothelium retains the ability to respond to NO, but is impaired in the signalling mechanisms activating nitric oxide synthase (NOS). Drugs that potentiate NO signalling may be effective in partially reversing the microcirculatory dysfunction after TBI. Studies are currently underway in rats using the MRI-Blood Oxygen Dependent signal (MRIBOLD) with hypercapnia challenge to assess CVRx.(Pronger et al, 2014)

Preclinical work with treatments that increase $C B F$ and angiogenesis

NO is the primary endogenous vasodilator in the brain. NO exerts its vasodilatory effects by activating soluble guanylyl cyclase, resulting in increased intracellular cyclic 
guanosine monophosphate (cGMP). cGMP is short lived, as it is rapidly hydrolyzed by cGMP phosphodiesterase (phosphodiesterase type 5, PDE5). Specific PDE5 inhibitors have come into widespread use in clinical medicine over the past 15 years. The first of these, sildenafil (Viagra ${ }^{\circledR}$ ), is approved by the FDA for the treatment of erectile dysfunction and primary pulmonary hypertension. Sildenafil enters the brain readily and has been extensively studied in animal models of CNS disease.(Atalay et al, 2006;Ding et al., 2008;Pifarre et al., 2010;Puzzo et al, 2009;Zhang et al., 2002)

In experimental models of ischemic stroke, administration of sildenafil after MCA occlusion enhances neurologic recovery, but does not reduce infarct size.(Zhang et al., 2002) Several studies have investigated the beneficial effect of sildenafil on the ischemic boundary area utilizing MRI methods to measure CBF (by ASL) and axonal remodelling (with diffusion tensor imaging).(Ding et al., 2008) Sildenafil produces a beneficial effect on neurological recovery possibly via increased local $\mathrm{CBF}$ as well as enhanced neurogenesis, angiogenesis, and axonal remodeling in the peri-infarct zone.(Ding et al., 2008;Li et al, 2007;Zhang et al., 2002)

Other FDA-approved medications have proven beneficial in preclinical trials potentially through angiogenesis promotion after TCVI, including atorvastatin(Wang et al, 2012) and glibenclamide.(Kurland et al, 2013) Both drugs have multiple modes of action. Glibenclamide has several protective effects in the CNS, including its action on cerebral microvessels to reduce edema formation and secondary haemorrhage, inhibition of cellular necrosis, potent antiinflammatory effects and neurogenesis promotion.(Kurland et al., 2013) In a rat model of CCI, glibenclamide administered shortly after injury, reduces the spatial and temporal "blossoming" 
of hemorrhagic contusion.(Simard et al, 2009) The mechanism is hypothesized to act through glibenclamide's inhibitory effects on Sur1, a sulfonylurea receptor that is upregulated in penumbral microvessels within hours after injury.(Patel et al, 2010;Simard et al., 2009) Likewise, atorvastatin appears to reduce secondary vascular injury after TBI through its antioxidataive and anti-inflammatory effects.(Wang et al., 2012) In addition, atorvastatin may be beneficial through its angiogenic effects. Atorvastatin increases mobilization of endothelial progenitor cells (EPCs) which promote vascular remodelling in animal models of ischemia and coronary heart disease.(Matsumura et al, 2009; Vasa et al, 2001) Atorvastatin treatment within 1 hour of FPI in rats followed by daily treatments for 14 days significantly increased circulating EPCs. The EPC increase was highly correlated with increased perilesional vascular density $(\mathrm{r}=$ 0.878) and was associated with improved functional measures.(Wang et al., 2012)

\section{Neuroimaging studies in humans}

Cerebral blood flow has been extensively studied after TBI in humans, mostly in the acute period within a few days of injury.(Furuya et al, 2003;Menon, 2006) Several investigators have also studied CBF weeks to years after TBI. There is a consistent body of literature in humans indicating that deficits in cerebral blood flow (CBF) are common after TBI, including repetitive mTBI.(Bonne et al, 2003) Bonne et al. used Technetium $\left({ }^{99 \mathrm{~m}} \mathrm{Tc}\right)$ hexamethylpropyleneamine oxime (Tc-99m-HMPAO) single photon emission computed tomography (SPECT) to measure regional CBF in symptomatic patients with chronic TBI. This study found several regions of hypoperfusion in the brains of symptomatic TBI subjects, particularly in the lateral and mesial frontal structures. The same group used a similar SPECT imaging technique before and after administration of acetazolamide and uncovered areas of 
abnormal perfusion that were not otherwise seen in mTBI.(Barkai et al, 2004) Using SPECT, Lewine et al (Lewine et al, 2007) identified abnormalities in $40 \%$ of mTBI patients with chronic symptoms ( $\mathrm{n}=30$ ). In this study SPECT was found to be significantly more sensitive than MRI in detecting abnormalities after mTBI. A recent systematic review of SPECT in the diagnosis of mTBI that analyzed 19 longitudinal and 52 cross-sectional studies, found Class IIa evidence that SPECT outperformed CT and MRI in the diagnosis of both acute and chronic TBI, especially with mTBI.(Raji et al, 2014) Ten of the longitudinal studies directly compared SPECT to CT or MRI results. In all 10 studies, SPECT identified lesions that were not seen by conventional imaging. The most common brain regions showing abnormalities by SPECT in cross-sectional studies were frontal (94\%) and temporal (77\%) lobes, followed by parietal, occipital and cerebellum. SPECT perfusion changes significantly correlated with neuropsychological or neurological deficits. Finally, Inoue et al (Inoue et al, 2005) used Xenon-CT to demonstrate persistent deficits in $\mathrm{CBF}$ in 55\% of patients with severe TBI persisting up to 6 months after injury.

Other advanced MRI techniques have been helpful in evaluating TCVI. ASL utilizes a radiofrequency pulse to "label" water protons in blood flowing through the carotid arteries by inverting their spins, and generating a contrast. The dissipation of the labeled spins in the distal cerebral vasculature is used as an indirect measure of CBF. A major advantage of ASL is that it provides a direct measurement of arterial perfusion in absolute units of $\mathrm{CBF}$ (cc/100 $\mathrm{gm} / \mathrm{min})$.(Edlow and $\mathrm{Wu}, 2012)$ ASL perfusion imaging studies have revealed alterations in global and regional resting CBF in TBI patients of all severities. Kim et al (Kim et al, 2010;Kim et al, 2012) showed that patients with chronic moderate-to-severe TBI have reduced global CBF in the resting state, as well as decreased regional perfusion in the thalamus, posterior cingulate 
cortex, and frontal cortex. Regions with decreased resting CBF also had altered task-related activation during an ASL fMRI working memory paradigm.(Kim et al., 2010;Kim et al., 2012) Regional relative CBF can also be calculated with perfusion-weighted imaging (PWI). A cohort of 15 symptomatic sports-related concussion patients studied a mean of 6 months after their concussion, shows reduced CBF in the thalami bilaterally compared to healthy age-matched controls.(Bartnik-Olson et al., 2014) They also exhibit reduced CBV in the left thalamus compared to healthy controls. These findings are thought to reflect chronic microvascular change that may underlie persistent post-concussive symptoms in the subjects. Susceptibilityweighted imaging (SWI) has been shown to detect more microbleeds than gradient recalled echo MRI (GRE) in patients with traumatic axonal injury.(Tong et al, 2004) The total number and volume of microbleeds detected by SWI correlate with functional outcomes after TBI. In a recent study of SWI imaging of mTBI patients in the subacute (8 to 60 days) stage after injury, traumatic microbleeds are seen in $23 \%$ of mTBI patients.(Huang et al, 2015) Presence of microbleeds detected by SWI in mTBI patients inversely correlate with digit span score.

Assessment of cerebrovascular reactivity in humans.

Well-established methods exist to study cerebrovascular reactivity non-invasively in humans, after stimuli such as hypercapnia, breath holding, or acetazolamide.(Kassner and Roberts, 2004) Transcranial Doppler (TCD) and MRI are the most popular methods in recent studies. TCD offers the advantage of very high temporal resolution, but suffers from poor spatial resolution. MRI is complementary with superb spatial resolution but poor temporal resolution. A large prospective study of 299 patients assessed the incidence of cerebral vasospasm via TCD. 
TCD was administered at regular intervals within the first two weeks after critical care hospitalization for moderate or severe TBI.(Oertel et al, 2005) Nearly half (45.2\%) of the patients had at least one TCD criterion for vasospasm. The highest risk of vasospasm occurred at day 3 after injury in the anterior circulation, and reflects the dynamic manifestations of microvascular dysfunction after TBI. CVRx, measured with both TCD and near infra-red spectroscopy (NIRS), was found to be decreased in professional boxers,(Bailey et al, 2013) particularly in boxers who had experienced the highest mTBI exposures. In this study, 12 boxers (mean age 27 years with mean 13 years competitive boxing and on average greater than 100 rounds boxed) were studied at minimum 72 hours after a boxing bout with both TCD and NIRS. Those assessments were coupled with dynamic challenges include hypercapnia, hypocapnia and orthostatic challenges. The group also underwent neuropsychological testing and the results were compared to 12 age/gender/fitness- matched healthy controls. The boxers had chronically impaired CVRx by all measures compared to the controls. Lower CVRx measurements correlate with worse neurocognitive dysfunction and was inversely correlated with head injury exposure. There also appeared to be a threshold minimum number of sparring matches corresponding to a reduced CVRx above which the boxers appeared to be more susceptible to persistent neurocognitive deficits on formal neuropsychological testing. A recent meta-analysis of over 2,000 articles identified 3 studies examining sports-related concussion and CVRx via TCD in 42 athletes (primarily boxers and hockey players) and 33 healthy controls. The athletes were all examined between 2 and 5 days after a sports-related concussion. All 3 studies found decreased CVRx in the acute period after a mTBI.(Gardner et al, 2014) CVRx via TCD is an active field of research. There are several ongoing studies of TCD coupled with breath-holding and other dynamic maneuvers in acute and chronic TBI. 
NIRS, another noninvasive measure of CVRx when coupled with dynamic challenges, is currently being assessed by several groups after TBI as well as other neurologic conditions.(Lee et al, 2009; Rodriguez Merzagora et al, 2014;Zweifel et al, 2010a;Zweifel et al, 2010b) Like TCD, NIRS offers a very high temporal resolution with good spatial resolution, albeit not as high as MRI. NIRS utilizes a near infrared emitting laser diode surrounded by multiple detecting photodiodes that measure light attenuation at different frequencies. Changes in CBF and associated changes in tissue concentrations of oxy- and deoxy-hemoglobin are measured by NIRS. NIRS allows for reliable CVRx measurements over time during dynamic challenges that are independent of hemoglobin concentration, skull thickness and extracranial circulation.(Kainerstorfer et al, 2014) Using this method, decreased CVRx results from increased blood transit time in the cerebral microvasculature and supports CVRx as an indirect measure of TCVI. Using a NIRS-based CVRx index in 40 acute TBI patients, total hemoglobin reactivity index (THx) measured via NIRS correlated with the intracranial pressure (ICP) derived cerebrovascular pressure reactivity index (PRx).(Zweifel et al., 2010b) This method is preferable in patients for whom invasive ICP monitoring was not feasible. A follow-on study of 37 TBI patients in the critical care unit showed a good correlation between THx measured via NIRS and ICP. This method could be used as a noninvasive assessment of CVRx in certain patients without ICP monitoring.(Diedler et al, 2011) Functional NIRS (fNIRS) testing has also been carried out in the chronic stage of TBI to assess chronic CVRx changes as a result of TBI. NIRS testing is performed with a cognitive load or vasodilatory stimulus to assess the ability of the cerebral microvasculature to respond to increased demand. CVRx was measured in a small study of 6 chronic moderate and severe TBI patients (mean 18 years after TBI) during a cognitive 
challenge. TBI patients showed significantly reduced CVRx compared to 15 age-gender matched controls. The CVRx abnormalities corresponded to performance on the cognitive tests.(Rodriguez Merzagora et al., 2014) CVRx can also be measured with fNIRS coupled with a hypercapnia challenge.

Hypercapnia-BOLD MRI reliably measures deficits in CVRx in the chronic stage after TBI.

The most important limitation of CBF studies in evaluating TCVI is that CBF is tightly coupled to cerebral metabolic demand. CBF measurements alone cannot distinguish between a deficit resulting from a primary vascular injury or from subtle neuronal or axonal injury. These injuries are too subtle to be detected by structural MRI yet sufficient to reduce cerebral metabolic demand. More direct measures of cerebral vascular injury require assessment of CVRx to manipulations that directly affect endothelial function. Such studies have recently become possible through advances in MRI technology. One method combines MRI with the Blood Oxygen Dependent (BOLD) signal in response to hypercapnia challenge. The hypercapnia challenge is administered during the MRI with the inhalation of room air alternating every minute with room air $+5 \% \mathrm{CO}_{2}$ over a period of 7 minutes. The CVRx is the ratio of change in BOLD signal to the change in end-tidal $\mathrm{CO}_{2}\left(\mathrm{EtCO}_{2}\right)$ and a CVRx map (Fig. 8) can be analysed for each voxel in the brain and correlated with the structural MRI images (Fig. 9).

Our group has been studying MRI-BOLD with hypercapnia challenge in patients in the chronic stage (> 6 months) after TBI (ClinicalTrials.gov NCT01762475). Preliminary results in 
27 patients (6 healthy controls and 21 TBI subjects) show significantly decreased CVRx in the chronic stage after moderate or severe TBI (Fig. 10).

We subsequently adapted this technique, MRI-BOLD with hypercapnia challenge, to the portable, less expensive, office-based NIRS technology. We assessed whether NIRS coupled with the hypercapnia challenge could be used to assess CVRx. fNIRS was studied in the same cohort of patients in the chronic stage (> 6 months) after TBI (ClinicalTrials.gov NCT01797549). Interim results have shown very similar reductions in CVRx in the TBI group, similar to that found with MRI-BOLD with a high degree of correlation (Fig. 11). This study supports the persistence of TCVI in the chronic stage of TBI and the ability to use NIRS as reliably as the more expensive MRI technique. Both technologies can be adapted to animal models for exploration of CVRx as a physiologic marker of TCVI in experimental TBI models. These technologies can also be a useful and much needed pharmacodynamic marker in therapeutic trials addressing the TCVI phenotype of TBI.

\section{CONCLUSIONS:}

Until recently, TCVI has been an under-studied component of TBI despite a robust theoretical construct and a large body of empirical evidence from clinical and preclinical studies. These studies support the near universal presence of TCVI after TBI. TCVI plays a potentially important role in chronic post-concussive symptoms and neurodegenerative disorders such as CTE manifesting remotely after TBI. Pathological studies have only recently focused on vascular injury after TBI, especially in chronic TBI. More work needs to be done to catalogue the neuropathological features associated with the various severities of TBI at from acute to 
chronic time periods after injury. Physiologic studies are critical to understanding the pathophysiology as they can record the evolution of TCVI over multiple time points after an injury in a dynamic fashion. As new technologies are developed to measure the function of the cerebral microvasculature, we will achieve a better understanding of the role of TCVI in clinical symptoms both acutely and remotely after TBI. Finally, understanding the pathophysiology of TCVI will aid in the development of effective treatments targeting the underlying pathology, and, likely serve as pharmacodynamic markers of therapeutic efficacy. 
Acknowledgements: Work in the authors' laboratory was supported by the Center for Neuroscience and Regenerative Medicine, and by the Chronic Effects of Neurotrauma Consortium. The contents of this paper are solely the responsibility of the authors and do not represent the official views of the Department of Defense or the Center for Neuroscience and Regenerative Medicine. 


\section{REFERENCES}

1. Adams, C.W., Bruton, C.J., 1989. The cerebral vasculature in dementia pugilistica. J Neurol Neurosurg. Psychiatry 52, 600-604.

2. Atalay, B., Caner, H., Cekinmez, M., Ozen, O., Celasun, B., Altinors, N., 2006. Systemic administration of phosphodiesterase $\mathrm{V}$ inhibitor, sildenafil citrate, for attenuation of cerebral vasospasm after experimental subarachnoid hemorrhage. Neurosurgery 59, 1102-1107.

3. Bailey, D.M., Jones, D.W., Sinnott, A., Brugniaux, J.V., New, K.J., Hodson, D., Marley, C.J., Smirl, J.D., Ogoh, S., Ainslie, P.N., 2013. Impaired cerebral haemodynamic function associated with chronic traumatic brain injury in professional boxers. Clin. Sci. (Lond) 124, 177-189.

4. Baranova, A.I., Wei, E.P., Ueda, Y., Sholley, M.M., Kontos, H.A., Povlishock, J.T., 2008. Cerebral vascular responsiveness after experimental traumatic brain injury: the beneficial effects of delayed hypothermia combined with superoxide dismutase administration. Journal of Neurosurgery 109, 502-509.

5. Barkai, G., Goshen, E., Tzila, Z.S., Dolberg, O.T., Pick, C.G., Bonne, O., Schreiber, S., 2004. Acetazolamide-enhanced neuroSPECT scan reveals functional impairment after minimal traumatic brain injury not otherwise discernible. Psychiatry Res. 132, 279-283.

6. Bartnik-Olson, B.L., Holshouser, B., Wang, H., Grube, M., Tong, K., Wong, V., Ashwal, S., 2014. Impaired neurovascular unit function contributes to persistent symptoms after concussion: a pilot study. J Neurotrauma 31, 1497-1506.

7. Besancon, E., Guo, S., Lok, J., Tymianski, M., Lo, E.H., 2008. Beyond NMDA and AMPA glutamate receptors: emerging mechanisms for ionic imbalance and cell death in stroke. Trends in Pharmacological Sciences 29, 268-275.

8. Bonne, O., Gilboa, A., Louzoun, Y., Kempf-Sherf, O., Katz, M., Fishman, Y., BenNahum, Z., Krausz, Y., Bocher, M., Lester, H., Chisin, R., Lerer, B., 2003. Cerebral blood flow in chronic symptomatic mild traumatic brain injury. Psychiatry Res. 124, 141152.

9. Breteler, M.M.B., 2000. Vascular risk factors for Alzheimer's disease: An epidemiologic perspective. Neurobiology of Aging 21, 153-160.

10. Castejon, O.J., 2011. Ultrastructural pathology of cortical capillary pericytes in human traumatic brain oedema. Folia Neuropathol. 49, 162-173.

11. Castejon, O.J., 2014. Ultrastructural alterations of human cortical capillary basement membrane in human brain oedema. Folia Neuropathol. 52, 10-21. 
12. Chabriat, H., Joutel, A., Dichgans, M., Tournier-Lasserve, E., Bousser, M.G., 2009. Cadasil. Lancet Neurol 8, 643-653.

13. Cherian, L., Goodman, J.C., Robertson, C., 2011. Improved cerebrovascular function and reduced histological damage with darbepoietin alfa administration after cortical impact injury in rats. J Pharmacol. Exp. Ther. 337, 451-456.

14. Chodobski, A., Zink, B.J., Szmydynger-Chodobska, J., 2011. Blood-brain barrier pathophysiology in traumatic brain injury. Transl. Stroke Res. 2, 492-516.

15. Cohen, Z., Bonvento, G., Lacombe, P., Hamel, E., 1996. Serotonin in the regulation of brain microcirculation. Prog. Neurobiol. 50, 335-362.

16. Corsellis, J.A., Brierley, J.B., 1959. Observations on the pathology of insidious dementia following head injury. J Ment. Sci. 105, 714-720.

17. Corsellis, J.A., Bruton, C.J., Freeman-Browne, D., 1973. The aftermath of boxing. Psychol. Med. 3, 270-303.

18. Danaila, L., Popescu, I., Pais, V., Riga, D., Riga, S., Pais, E., 2013. Apoptosis, paraptosis, necrosis, and cell regeneration in posttraumatic cerebral arteries. Chirurgia. (Bucur. ) 108, 319-324.

19. del Zoppo, G.J., 2013. Toward the neurovascular unit. A journey in clinical translation: 2012 Thomas Willis Lecture. Stroke 44, 263-269.

20. Dewitt, D.S., Prough, D.S., 2003. Traumatic cerebral vascular injury: The effects of concussive brain injury on the cerebral vasculature. J. Neurotrauma 20,795-825.

21. Diaz-Arrastia, R., Kochanek, P.M., Bergold, P., Kenney, K., Marx, C., Grimes, J., Loh, Y., Adam, G., Oskvig, D.B., Curley, K., Salzer, W., 2013. Pharmacotherapy of Traumatic Brain Injury: State of the Science and the Road Forward Report of the Department of Defense Neurotrauma Pharmacology Workgroup. J Neurotrauma.

22. Diedler, J., Zweifel, C., Budohoski, K.P., Kasprowicz, M., Sorrentino, E., Haubrich, C., Brady, K.M., Czosnyka, M., Pickard, J.D., Smielewski, P., 2011. The limitations of nearinfrared spectroscopy to assess cerebrovascular reactivity: the role of slow frequency oscillations. Anesth. Analg. 113, 849-857.

23. Dietrich, W.D., Alonso, O., Halley, M., 1994. Early microvascular and neuronal consequences of traumatic brain injury: a light and electron microscopic study in rats. J Neurotrauma 11, 289-301.

24. Dimmeler, S., Haendeler, J., Nehls, M., Zeiher, A.M., 1997. Suppression of apoptosis by nitric oxide via inhibition of interleukin-1beta-converting enzyme (ICE)-like and cysteine protease protein (CPP)-32-like proteases. J Exp. Med. 185, 601-607. 
25. Ding, G., Jiang, Q., Li, L., Zhang, L., Zhang, Z.G., Ledbetter, K.A., Panda, S., Davarani, S.P., Athiraman, H., Li, Q., Ewing, J.R., Chopp, M., 2008. Magnetic resonance imaging investigation of axonal remodeling and angiogenesis after embolic stroke in sildenafiltreated rats. J. Cereb. Blood Flow Metab 28, 1440-1448.

26. Doppenberg, E.M.R., Bullock, R., 1997. Clinical neuroprotective trials in severe traumatic brain injury: Lessons from previous studies. J. Neurotrauma 14, 71-80.

27. Dore-Duffy, P., Owen, C., Balabanov, R., Murphy, S., Beaumont, T., Rafols, J.A., 2000. Pericyte migration from the vascular wall in response to traumatic brain injury. Microvasc. Res. 60, 55-69.

28. Edlow, B.L., Wu, O., 2012. Advanced neuroimaging in traumatic brain injury. Semin. Neurol 32, 374-400.

29. Ellison, M.D., Erb, D.E., Kontos, H.A., Povlishock, J.T., 1989. Recovery of impaired endothelium-dependent relaxation after fluid-percussion brain injury in cats. Stroke 20, 911-917.

30. Furuya, Y., Hlatky, R., Valadka, A.B., Diaz, P., Robertson, C.S., 2003. Comparison of cerebral blood flow in computed tomographic hypodense areas of the brain in headinjured patients. Neurosurgery 52, 340-346.

31. Gao, G., Oda, Y., Wei, E.P., Povlishock, J.T., 2010. The adverse pial arteriolar and axonal consequences of traumatic brain injury complicated by hypoxia and their therapeutic modulation with hypothermia in rat. J. Cereb. Blood Flow Metab 30, 628637.

32. Gardner, A.J., Tan, C.O., Ainslie, P.N., van, D.P., Stanwell, P., Levi, C.R., Iverson, G.L., 2014. Cerebrovascular reactivity assessed by transcranial Doppler ultrasound in sportrelated concussion: a systematic review. Br. J Sports Med.

33. Giza, C.C., Hovda, D.A., 2014. The new neurometabolic cascade of concussion. Neurosurgery 75 Suppl 4, S24-S33.

34. Glushakova, O.Y., Johnson, D., Hayes, R.L., 2014. Delayed increases in microvascular pathology after experimental traumatic brain injury are associated with prolonged inflammation, blood-brain barrier disruption, and progressive white matter damage. J Neurotrauma 31, 1180-1193.

35. Golding, E.M., 2002. Sequalae following traumatic brain injury. The cerebrovascular perspective. Brain Research Rev. 38, 377-388.

36. Golding, E.M., Robertson, C.S., Bryan, R.M., 1999. The consequences of traumatic brain injury on cerebral blood flow and autoregulation: a review. Clin Exp Hypertens 21, 299332. 
37. Goldstein, L.E., Fisher, A.M., Tagge, C.A., Zhang, X.L., Velisek, L., Sullivan, J.A., Upreti, C., Kracht, J.M., Ericsson, M., Wojnarowicz, M.W., Goletiani, C.J., Maglakelidze, G.M., Casey, N., Moncaster, J.A., Minaeva, O., Moir, R.D., Nowinski, C.J., Stern, R.A., Cantu, R.C., Geiling, J., Blusztajn, J.K., Wolozin, B.L., Ikezu, T., Stein, T.D., Budson, A.E., Kowall, N.W., Chargin, D., Sharon, A., Saman, S., Hall, G.F., Moss, W.C., Cleveland, R.O., Tanzi, R.E., Stanton, P.K., McKee, A.C., 2012. Chronic traumatic encephalopathy in blast-exposed military veterans and a blast neurotrauma mouse model. Sci. Transl. Med. 4, 134ra60.

38. Graham, D.I., Gennarelli, T.A., McIntosh, T.K., 2002. Trauma. In: Graham, D.I., McKenzie, J.E. (Eds.), Greenfield's Neuropathology Edward Arnold, London, pp. 823898.

39. Hamel, E., 2006. Perivascular nerves and the regulation of cerebrovascular tone. J Appl. Physiol (1985. ) 100, 1059-1064.

40. Hausmann, R., Betz, P., 2000. The time course of the vascular response to human brain injury--an immunohistochemical study. Int. J Legal Med. 113, 288-292.

41. Hawkins, B.T., Davis, T.P., 2005. The blood-brain barrier/neurovascular unit in health and disease. Pharmacol. Rev. 57, 173-185.

42. Hayward, N.M., Tuunanen, P.I., Immonen, R., Ndode-Ekane, X.E., Pitkanen, A., Grohn, O., 2011. Magnetic resonance imaging of regional hemodynamic and cerebrovascular recovery after lateral fluid-percussion brain injury in rats. J Cereb. Blood Flow Metab 31, $166-177$.

43. Huang, Y.L., Kuo, Y.S., Tseng, Y.C., Chen, D.Y., Chiu, W.T., Chen, C.J., 2015. Susceptibility-weighted MRI in mild traumatic brain injury. Neurology 84, 580-585.

44. Hyder, A.A., Wunderlich, C.A., Puvanachandra, P., Gururaj, G., Kobusingye, O.C., 2007. The impact of traumatic brain injuries: a global perspective. NeuroRehabilitation. 22, 341-353.

45. Inoue, M., McHugh, M., Pappius, H.M., 1991. The effect of alpha-adrenergic receptor blockers prazosin and yohimbine on cerebral metabolism and biogenic amine content of traumatized brain. J Cereb. Blood Flow Metab 11, 242-252.

46. Inoue, Y., Shiozaki, T., Tasaki, O., Hayakata, T., Ikegawa, H., Yoshiya, K., Fujinaka, T., Tanaka, H., Shimazu, T., Sugimoto, H., 2005. Changes in cerebral blood flow from the acute to the chronic phase of severe head injury. J. Neurotrauma 22, 1411-1418.

47. Kainerstorfer, J.M., Sassaroli, A., Hallacoglu, B., Pierro, M.L., Fantini, S., 2014. Practical steps for applying a new dynamic model to near-infrared spectroscopy measurements of hemodynamic oscillations and transient changes: implications for cerebrovascular and functional brain studies. Acad. Radiol. 21, 185-196. 
48. Kassner, A., Roberts, T.P., 2004. Beyond perfusion: cerebral vascular reactivity and assessment of microvascular permeability. Top. Magn Reson. Imaging 15, 58-65.

49. Kim, J., Whyte, J., Patel, S., Avants, B., Europa, E., Wang, J., Slattery, J., Gee, J.C., Coslett, H.B., Detre, J.A., 2010. Resting cerebral blood flow alterations in chronic traumatic brain injury: an arterial spin labeling perfusion FMRI study. J Neurotrauma 27, 1399-1411.

50. Kim, J., Whyte, J., Patel, S., Europa, E., Slattery, J., Coslett, H.B., Detre, J.A., 2012. A perfusion fMRI study of the neural correlates of sustained-attention and working-memory deficits in chronic traumatic brain injury. Neurorehabil. Neural Repair 26, 870-880.

51. Kontos, H.A., Dietrich, W.D., Wei, E.P., Ellis, E.F., Povlishock, J.T., 1980. Abnormalities of the cerebral microcirculation after traumatic injury: the relationship of hypertension and prostaglandins. Advances in experimental medicine and biology 131, 243-256.

52. Kurland, D.B., Tosun, C., Pampori, A., Karimy, J.K., Caffes, N.M., Gerzanich, V., Simard, J.M., 2013. Glibenclamide for the treatment of acute CNS injury. Pharmaceuticals. (Basel) 6, 1287-1303.

53. Lassen, N.A., 1959. Cerebral blood flow and oxygen consumption in man. Physiol Rev. $39,183-238$.

54. Lecrux, C., Hamel, E., 2011. The neurovascular unit in brain function and disease. Acta Physiol (Oxf) 203, 47-59.

55. Lee, J.K., Kibler, K.K., Benni, P.B., Easley, R.B., Czosnyka, M., Smielewski, P., Koehler, R.C., Shaffner, D.H., Brady, K.M., 2009. Cerebrovascular reactivity measured by near-infrared spectroscopy. Stroke 40, 1820-1826.

56. Lenzlinger, P.M., Marx, A., Trentz, O., Kossmann, T., Morganti-Kossmann, M.C., 2002. Prolonged intrathecal release of soluble Fas following severe traumatic brain injury in humans. J Neuroimmunol. 122, 167-174.

57. Lewine, J.D., Davis, J.T., Bigler, E.D., Thoma, R., Hill, D., Funke, M., Sloan, J.H., Hall, S., Orrison, W.W., 2007. Objective documentation of traumatic brain injury subsequent to mild head trauma: multimodal brain imaging with MEG, SPECT, and MRI. J. Head Trauma Rehabil. 22, 141-155.

58. Li, L., Jiang, Q., Zhang, L., Ding, G., Gang, Z.Z., Li, Q., Ewing, J.R., Lu, M., Panda, S., Ledbetter, K.A., Whitton, P.A., Chopp, M., 2007. Angiogenesis and improved cerebral blood flow in the ischemic boundary area detected by MRI after administration of sildenafil to rats with embolic stroke. Brain Research 1132, 185-192.

59. Marchesi, V.T., 2014. Alzheimer's disease and CADASIL are heritable, adult-onset dementias that both involve damaged small blood vessels. Cell Mol. Life Sci. 71, 949955. 
60. Marklund, N., Bakshi, A., Castelbuono, D.J., Conte, V., McIntosh, T.K., 2006. Evaluation of pharmacological treatment strategies in traumatic brain injury. Curr. Pharm. Des 12, 1645-1680.

61. Martland, H., 1928. Dementia Pugilistica. JAMA 91, 1103-1107.

62. Matsumura, M., Fukuda, N., Kobayashi, N., Umezawa, H., Takasaka, A., Matsumoto, T., Yao, E.H., Ueno, T., Negishi, N., 2009. Effects of atorvastatin on angiogenesis in hindlimb ischemia and endothelial progenitor cell formation in rats. $\mathbf{J}$ Atheroscler. Thromb. 16, 319-326.

63. Maxwell, W.L., Irvine, A., Adams, J.H., Graham, D.I., Gennarelli, T.A., 1988. Response of cerebral microvasculature to brain injury. J Pathol. 155, 327-335.

64. McIntosh, T.K., 1993. Novel pharmacologic therapies in the treatment of experimental brain injury: A review. J. Neurotrauma 10, 215-261.

65. McIntosh, T.K., Juhler, M., Wieloch, T., 1998. Novel pharmacologic strategies in the treatment of experimental brain injury: 1998. J. Neurotrauma 15, 731-769.

66. McKee, A.C., Daneshvar, D.H., Alvarez, V.E., Stein, T.D., 2014. The neuropathology of sport. Acta Neuropathol. 127, 29-51.

67. McKee, A.C., Robinson, M.E., 2014. Military-related traumatic brain injury and neurodegeneration. Alzheimers Dement. 10, S242-S253.

68. McKee, A.C., Stein, T.D., Nowinski, C.J., Stern, R.A., Daneshvar, D.H., Alvarez, V.E., Lee, H.S., Hall, G., Wojtowicz, S.M., Baugh, C.M., Riley, D.O., Kubilus, C.A., Cormier, K.A., Jacobs, M.A., Martin, B.R., Abraham, C.R., Ikezu, T., Reichard, R.R., Wolozin, B.L., Budson, A.E., Goldstein, L.E., Kowall, N.W., Cantu, R.C., 2013. The spectrum of disease in chronic traumatic encephalopathy. Brain 136, 43-64.

69. Menon, D.K., 2006. Brain ischaemia after traumatic brain injury: lessons from $15 \mathrm{O} 2$ positron emission tomography. Curr. Opin. Crit Care 12, 85-89.

70. Narayan, R.K., Michel, M.E., The Clinical Trials in Head Injury Study Group, 2002. Clinical trials in head injury. J. Neurotrauma 19, 503-557.

71. Nicolakakis, N., Aboulkassim, T., Aliaga, A., Tong, X.K., Rosa-Neto, P., Hamel, E., 2011. Intact memory in TGF-beta1 transgenic mice featuring chronic cerebrovascular deficit: recovery with pioglitazone. J Cereb. Blood Flow Metab 31, 200-211.

72. Nicolakakis, N., Hamel, E., 2011. Neurovascular function in Alzheimer's disease patients and experimental models. J Cereb. Blood Flow Metab 31, 1354-1370.

73. Oertel, M., Boscardin, W.J., Obrist, W.D., Glenn, T.C., McArthur, D.L., Gravori, T., Lee, J.H., Martin, N.A., 2005. Posttraumatic vasospasm: the epidemiology, severity, and time 
course of an underestimated phenomenon: a prospective study performed in 299 patients. Journal of Neurosurgery 103, 812-824.

74. Olesen, J., Paulson, O.B., Lassen, N.A., 1971. Regional cerebral blood flow in man determined by the initial slope of the clearance of intra-arterially injected 133Xe. Stroke 2, 519-540.

75. Oppenheimer, D.R., 1968. Microscopic lesions in the brain following head injury. J Neurol Neurosurg. Psychiatry 31, 299-306.

76. Pappius, H.M., Dadoun, R., McHugh, M., 1988. The effect of p-chlorophenylalanine on cerebral metabolism and biogenic amine content of traumatized brain. J Cereb. Blood Flow Metab 8, 324-334.

77. Park, E., Bell, J.D., Siddiq, I.P., Baker, A.J., 2009. An analysis of regional microvascular loss and recovery following two grades of fluid percussion trauma: a role for hypoxiainducible factors in traumatic brain injury. J. Cereb. Blood Flow Metab 29, 575-584.

78. Pasco, A., Lemaire, L., Franconi, F., Lefur, Y., Noury, F., Saint-Andre, J.P., Benoit, J.P., Cozzone, P.J., Le Jeune, J.J., 2007. Perfusional deficit and the dynamics of cerebral edemas in experimental traumatic brain injury using perfusion and diffusion-weighted magnetic resonance imaging. J Neurotrauma 24, 1321-1330.

79. Patel, A.D., Gerzanich, V., Geng, Z., Simard, J.M., 2010. Glibenclamide reduces hippocampal injury and preserves rapid spatial learning in a model of traumatic brain injury. J. Neuropathol. Exp. Neurol. 69, 1177-1190.

80. Pifarre, P., Prado, J., Giralt, M., Molinero, A., Hidalgo, J., Garcia, A., 2010. Cyclic GMP phosphodiesterase inhibition alters the glial inflammatory response, reduces oxidative stress and cell death and increases angiogenesis following focal brain injury. J. Neurochem. 112, 807-817.

81. Pronger, A.M., Korotcov, A., Bosomtwi, A., Jones, S., Selwyn, R.G., Diaz-Arrastia, R. Multimodal neuroimaging with hypercapnia to monitor cerebrovascular function after traumatic brain injury and evaluate treatment. J Neurotrauma 31, A2-06. 2014.

Ref Type: Abstract

82. Puzzo, D., Staniszewski, A., Deng, S.X., Privitera, L., Leznik, E., Liu, S., Zhang, H., Feng, Y., Palmeri, A., Landry, D.W., Arancio, O., 2009. Phosphodiesterase 5 inhibition improves synaptic function, memory, and amyloid-beta load in an Alzheimer's disease mouse model. J. Neurosci. 29, 8075-8086.

83. Raji, C.A., Tarzwell, R., Pavel, D., Schneider, H., Uszler, M., Thornton, J., van, L.M., Cohen, P., Amen, D.G., Henderson, T., 2014. Clinical utility of SPECT neuroimaging in the diagnosis and treatment of traumatic brain injury: a systematic review. PLoS. One. 9, e91088. 
84. Rodriguez Merzagora, A.C., Izzetoglu, M., Onaral, B., Schultheis, M.T., 2014. Verbal working memory impairments following traumatic brain injury: an fNIRS investigation. Brain Imaging Behav. 8, 446-459.

85. Rodriguez-Baeza, A., Reina-de la Torre, F., Poca, A., Marti, M., Garnacho, A., 2003. Morphological features in human cortical brain microvessels after head injury: a threedimensional and immunocytochemical study. Anat. Rec. A Discov. Mol. Cell Evol. Biol. 273, 583-593.

86. Roy, C.S., Sherrington, C.S., 1890. On the Regulation of the Blood-supply of the Brain. J Physiol 11, 85-158.

87. Saatman, K.E., Duhaime, A.C., Bullock, R., Maas, A.I., Valadka, A., Manley, G.T., 2008. Classification of traumatic brain injury for targeted therapies. J. Neurotrauma 25, 719-738.

88. Sangiorgi, S., De, B.A., Protasoni, M., Manelli, A., Reguzzoni, M., Cividini, A., Dell'orbo, C., Tomei, G., Balbi, S., 2013. Early-stage microvascular alterations of a new model of controlled cortical traumatic brain injury: 3D morphological analysis using scanning electron microscopy and corrosion casting. J Neurosurg. 118, 763-774.

89. Schwarzmaier, S.M., Kim, S.W., Trabold, R., Plesnila, N., 2010. Temporal profile of thrombogenesis in the cerebral microcirculation after traumatic brain injury in mice. $\mathbf{J}$ Neurotrauma 27, 121-130.

90. Shlosberg, D., Benifla, M., Kaufer, D., Friedman, A., 2010. Blood-brain barrier breakdown as a therapeutic target in traumatic brain injury. Nat. Rev. Neurol 6, 393-403.

91. Simard, J.M., Kilbourne, M., Tsymbalyuk, O., Tosun, C., Caridi, J., Ivanova, S., Keledjian, K., Bochicchio, G., Gerzanich, V., 2009. Key role of sulfonylurea receptor 1 in progressive secondary hemorrhage after brain contusion. J. Neurotrauma 26, 22572267.

92. Sokoloff, L., 1981. Localization of functional activity in the central nervous system by measurement of glucose utilization with radioactive deoxyglucose. J Cereb. Blood Flow Metab 1, 7-36.

93. Stanimirovic, D.B., Friedman, A., 2012. Pathophysiology of the neurovascular unit: disease cause or consequence? J Cereb. Blood Flow Metab 32, 1207-1221.

94. Stein, S.C., Chen, X.H., Sinson, G.P., Smith, D.H., 2002. Intravascular coagulation: a major secondary insult in nonfatal traumatic brain injury. Journal of Neurosurgery 97, $1373-1377$.

95. Stein, S.C., Graham, D.I., Chen, X.H., Smith, D.H., 2004. Association between intravascular microthrombosis and cerebral ischemia in traumatic brain injury. Neurosurgery 54, 687-691. 
96. Suehiro, E., Ueda, Y., Wei, E.P., Kontos, H.A., Povlishock, J.T., 2003. Posttraumatic hypothermia followed by slow rewarming protects the cerebral microcirculation. J Neurotrauma 20, 381-390.

97. Tan, C.O., Meehan, W.P., III, Iverson, G.L., Taylor, J.A., 2014. Cerebrovascular regulation, exercise, and mild traumatic brain injury. Neurology 83, 1665-1672.

98. Thomale, U.W., Kroppenstedt, S.N., Beyer, T.F., Schaser, K.D., Unterberg, A.W., Stover, J.F., 2002. Temporal profile of cortical perfusion and microcirculation after controlled cortical impact injury in rats. J Neurotrauma 19, 403-413.

99. Tong, K.A., Ashwal, S., Holshouser, B.A., Nickerson, J.P., Wall, C.J., Shutter, L.A., Osterdock, R.J., Haacke, E.M., Kido, D., 2004. Diffuse axonal injury in children: Clinical correlation with hemorrhagic lesions. Annals of Neurology 56, 36-50.

100. Ueda, Y., Wei, E.P., Kontos, H.A., Suehiro, E., Povlishock, J.T., 2003. Effects of delayed, prolonged hypothermia on the pial vascular response after traumatic brain injury in rats. J Neurosurg. 99, 899-906.

101. Vasa, M., Fichtlscherer, S., Aicher, A., Adler, K., Urbich, C., Martin, H., Zeiher, A.M., Dimmeler, S., 2001. Number and migratory activity of circulating endothelial progenitor cells inversely correlate with risk factors for coronary artery disease. Circulation Research 89, E1-E7.

102. Villringer, A., Dirnagl, U., 1995. Coupling of brain activity and cerebral blood flow: basis of functional neuroimaging. Cerebrovasc. Brain Metab Rev. 7, 240-276.

103. Wang, B., Sun, L., Tian, Y., Li, Z., Wei, H., Wang, D., Yang, Z., Chen, J., Zhang, J., Jiang, R., 2012. Effects of atorvastatin in the regulation of circulating EPCs and angiogenesis in traumatic brain injury in rats. J Neurol Sci. 319, 117-123.

104. Wang, J., Shi, Y., Zhang, L., Zhang, F., Hu, X., Zhang, W., Leak, R.K., Gao, Y., Chen, L., Chen, J., 2014. Omega-3 polyunsaturated fatty acids enhance cerebral angiogenesis and provide long-term protection after stroke. Neurobiol. Dis. 68, 91-103.

105. Wei, E.P., Dietrich, W.D., Povlishock, J.T., Navari, R.M., Kontos, H.A., 1980. Functional, morphological, and metabolic abnormalities of the cerebral microcirculation after concussive brain injury in cats. Circulation Research 46, 37-47.

106. Wei, E.P., Hamm, R.J., Baranova, A.I., Povlishock, J.T., 2009. The long-term microvascular and behavioral consequences of experimental traumatic brain injury after hypothermic intervention. J. Neurotrauma 26, 527-537.

107. Wu, H., Jiang, H., Lu, D., Qu, C., Xiong, Y., Zhou, D., Chopp, M., Mahmood, A., 2011. Induction of angiogenesis and modulation of vascular endothelial growth factor receptor2 by simvastatin after traumatic brain injury. Neurosurgery 68, 1363-1371. 
108. Xing, C., Hayakawa, K., Lok, J., Arai, K., Lo, E.H., 2012. Injury and repair in the neurovascular unit. Neurol Res. 34, 325-330.

109. Xiong, Y., Mahmood, A., Meng, Y., Zhang, Y., Qu, C., Schallert, T., Chopp, M., 2010. Delayed administration of erythropoietin reducing hippocampal cell loss, enhancing angiogenesis and neurogenesis, and improving functional outcome following traumatic brain injury in rats: comparison of treatment with single and triple dose. J Neurosurg. $113,598-608$.

110. Yu, G.X., Mueller, M., Hawkins, B.E., Mathew, B.P., Parsley, M.A., Vergara, L.A., Hellmich, H.L., Prough, D.S., Dewitt, D.S., 2014. Traumatic brain injury in vivo and in vitro contributes to cerebral vascular dysfunction through impaired gap junction communication between vascular smooth muscle cells. J Neurotrauma 31, 739-748.

111. Zhang, R., Wang, Y., Zhang, L., Zhang, Z., Tsang, W., Lu, M., Zhang, L., Chopp, M., 2002. Sildenafil (Viagra) induces neurogenesis and promotes functional recovery after stroke in rats. Stroke 33, 2675-2680.

112. Zlokovic, B.V., 2008. The blood-brain barrier in health and chronic neurodegenerative disorders. Neuron 57, 178-201.

113. Zweifel, C., Castellani, G., Czosnyka, M., Carrera, E., Brady, K.M., Kirkpatrick, P.J., Pickard, J.D., Smielewski, P., 2010a. Continuous assessment of cerebral autoregulation with near-infrared spectroscopy in adults after subarachnoid hemorrhage. Stroke 41, 1963-1968.

114. Zweifel, C., Castellani, G., Czosnyka, M., Helmy, A., Manktelow, A., Carrera, E., Brady, K.M., Hutchinson, P.J., Menon, D.K., Pickard, J.D., Smielewski, P., 2010b. Noninvasive monitoring of cerebrovascular reactivity with near infrared spectroscopy in head-injured patients. J Neurotrauma 27, 1951-1958. 
Table 1. Summary of major components of traumatic vascular injury (TVI)

\begin{tabular}{|c|c|c|c|}
\hline \multicolumn{4}{|c|}{ Major Components of Acute Traumatic Vascular Injury (TVI) } \\
\hline Category of injury & Examples & Effect & Reference \\
\hline \multirow[t]{2}{*}{ Morphologic injury } & Endothelial lesion & Increased & Wei et al. 1980 \\
\hline & Endothelial barrier antigen & Decreased & Lin et al. 2001 \\
\hline \multirow[t]{5}{*}{ Physiologic injury } & Vascular reactivity & Decreased & Martin et al. 1997 \\
\hline & Vasoconstriction & Increased & Golding et al 1999 \\
\hline & Vasodilation & Decreased & Golding et al 1999 \\
\hline & BBB damage & Increased & Tanno et al.1992 \\
\hline & $\mathrm{O}_{2}$ consumption & Decreased & Wei et al. 1980 \\
\hline \multirow[t]{3}{*}{ Biochemical/molecular injury } & ${ }_{\mathrm{i}} \mathrm{NOS} / \mathrm{e} \mathrm{NOS}$ expression & Increased & Gahm et al. 2000 \\
\hline & Endothelial cell activation & Increased & Balabanov et al. 2001 \\
\hline & $\begin{array}{l}\text { von Willebrand factor } \\
\text { activation }\end{array}$ & Increased & Yokota et al. 2002 \\
\hline
\end{tabular}


Figure 1. A: Normal microvascular density in a $2 \mathrm{~mm}$ thick cortical sample with silicone perfusion technique. B: Representative silicone perfusion samples from sham (top), 2 atm FPI (middle) at 24 hours and 14 days after injury, and 3 atm FPI (bottom) at 24 hours and 14 days after injury, showing acute loss of microvasculature and abnormal regrowth at 2 weeks.

Ref: Park E, Bell JD, Siddiq IP, Baker AJ. An analysis of regional microvascular loss and recovery following two grades of fluid percussion trauma: a role for hypoxiainducible factors in traumatic brain injury. J Cereb Blood Flow Metab 2009;29:575-584.
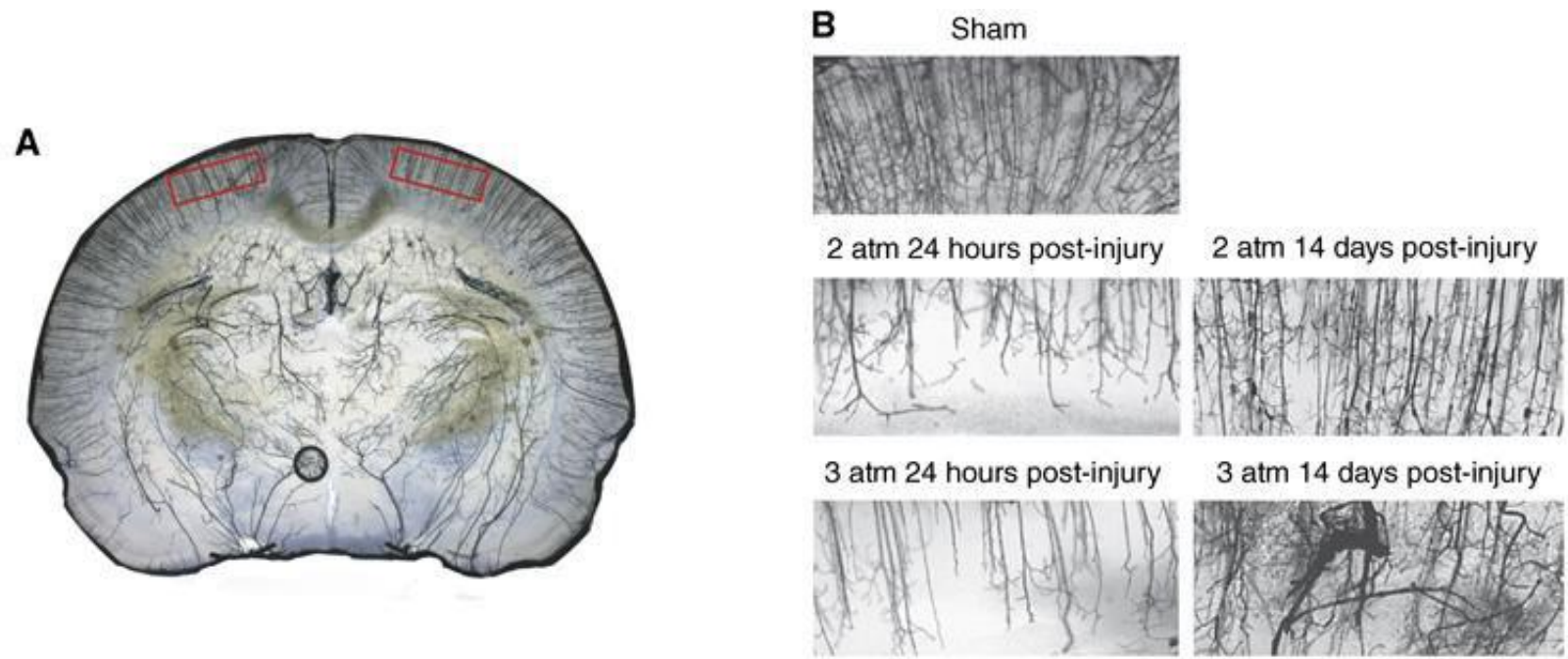
Figure 2. Microvascular changes 3 hours after controlled cortical impact (CCI). A: Scanning electronic micrograph $(\mathrm{SEM})$ of a corrosion cast from a control specimen $(*=$ pial vessel; \# $=$ perforating vessel). B: SEM from an experimental animal 3 hours after CCI with proximal constriction (\#) and swelling (*) of perforating vessels. C: Photomicrograph of light microscopy of same area with swelling (*) labeled. D: SEM area around fusiform swelling in B showing extravasations, evident as plastic rings (arrow) around vessel. E: Transmission electron microscopy (TEM) of extravasations seen in D. F: Photomicrograph of perforating vessel showing red blood cell (RBC) extravasation into the perivascular space $(*)$ outside endothelial cell wall. G: Vascular engorgement with red blood cells encased in extravasated resin (*). H: Transmission EM with RBCs entrapped within a perforating vessel (*). I: Accumulation of RBCs in the lumen of a cortical vessel (*).

Ref: Sangiorgi S, de Benedictis A, Protasoni M, et al. Early-stage microvascular alterations of a new model of controlled cortical traumatic brain inuury: 3D morphological analysis using scanning electron microscopy and corrosion casting. J Neurosurg 2013; 118(4):763-774.

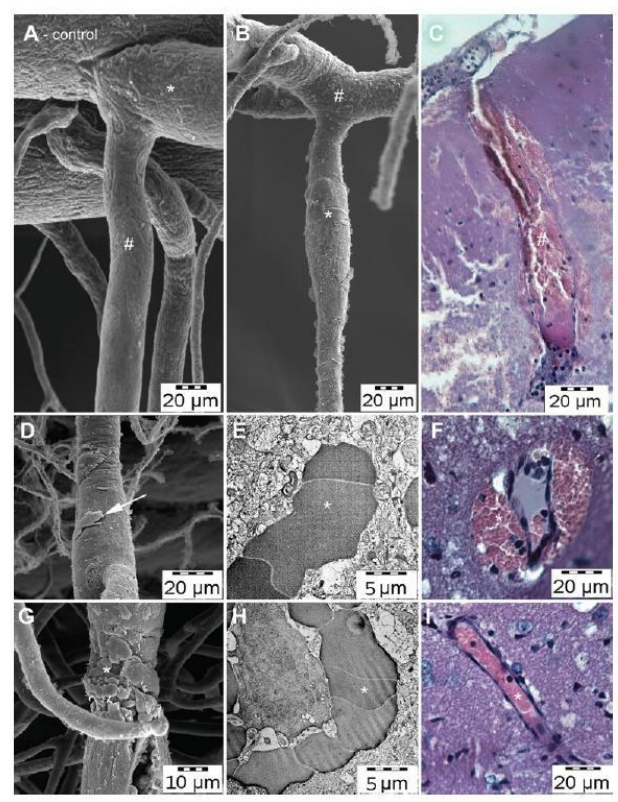


Figure 3. Microvascular changes 12 hours after CCI/experimental TBI. A: High magnification SEM of a cast of a perforating vessel with a distal constriction (arrow) below a cortical swelling (*) and grooves from endothelial cell imprints (\#). B: SEM of a cast of a perforating vessel with cortical capillaries removed $(*=$ swelling; $\#=$ endothelial cell imprints). C. Transmission EM of a swollen endothelial cell (*). D: Photomicrograph of swollen endothelial cell (*). E: High magnification SEM of a subcortical segment of a perforating vessel with imprints of swollen endothelial cells on the cast (arrow).

Ref: Sangiorgi S, de Benedictis A, Protasoni M, et al. Early-stage microvascular alterations of a new model of controlled cortical traumatic brain inury: 3D morphological analysis using scanning electron microscopy and corrosion casting. J Neurosurg 2013; 118(4):763-774.
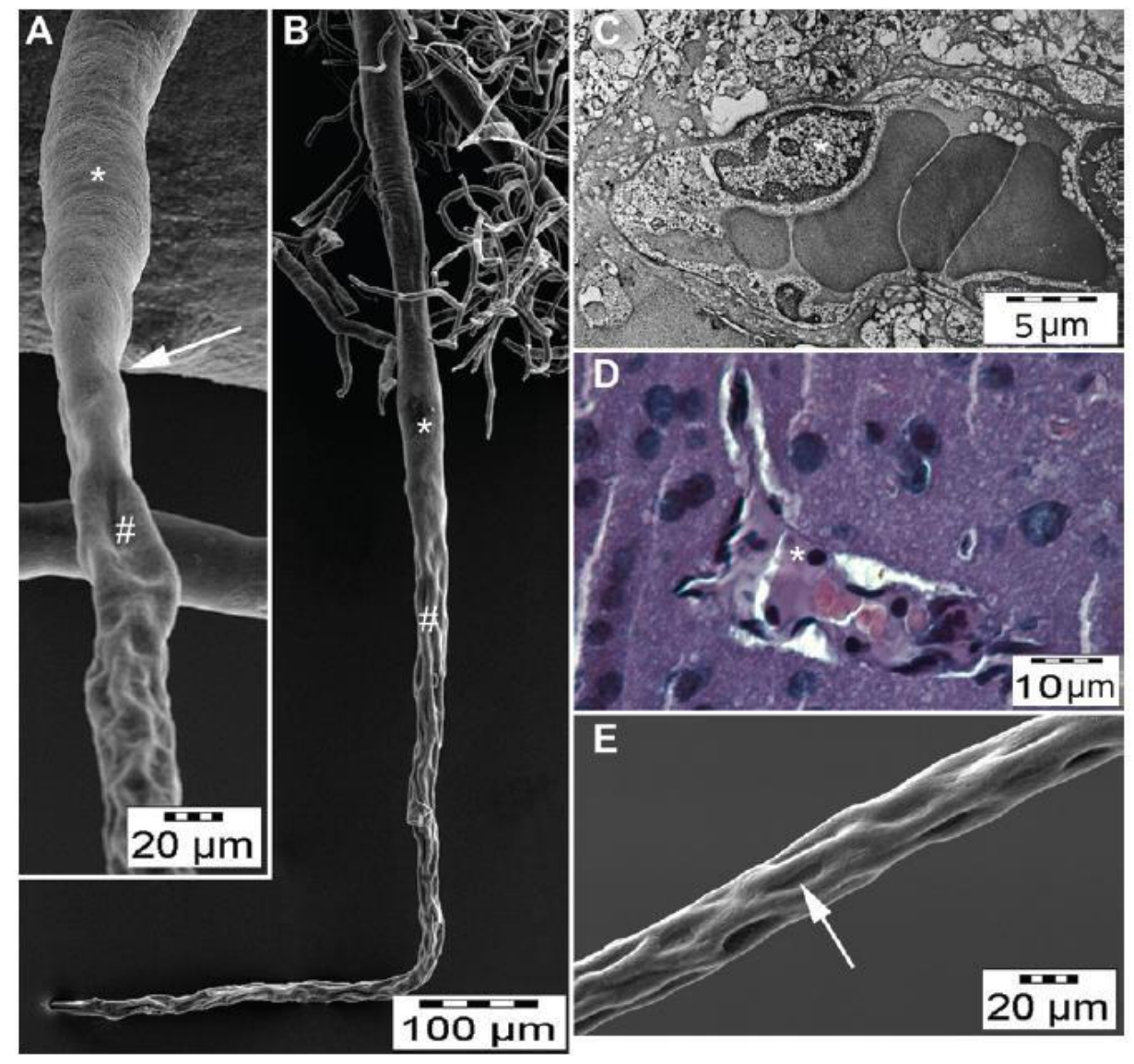
Figure 4. a: Gross section from a fatal case of TBI- an 18 year old who died at the scene of a vehicular accident- showing diffuse vascular injury with central diffuse axonal injury and peripheral cortical contusions.; b.-d: Examples of MRI sequences showing diffuse vascular injury after acute traumatic brain injury: b: Susceptibility weighted imaging (SWI) showing linear perivascular hemorrhages in the left inferior temporal lobe; c. SWI showing linear perivascular hemorrhages in the subcortical white matter of the left parietal lobe;

d. Fluid-attenuated inversion recovery (FLAIR) MRI showing bright area of linear hemorrhage in the left subcortical white matter at the frontoparietal junction.

Gross Pathology (a), Courtesy of Willie Stewart, M.D., Consultant Neuropathologist, Southern General Hospital, Glasgow, UK

MRIs (b-d), Courtesy of Larry Latour, Ph.D., CNRM, NINDS, NIH

a.

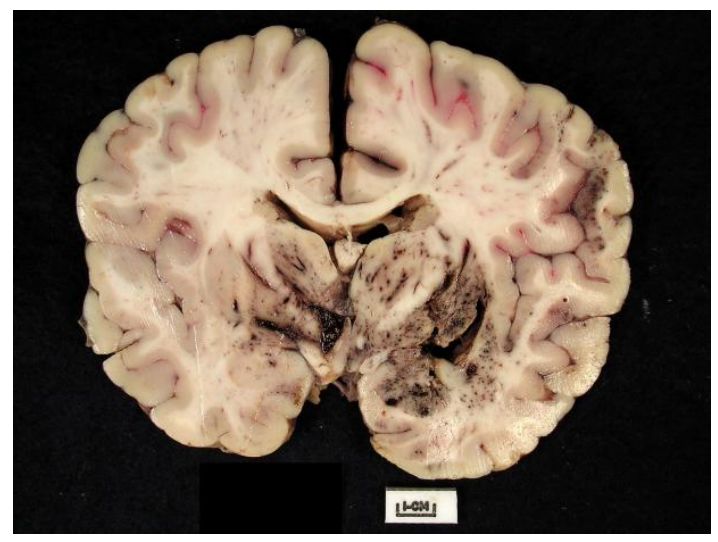

b.
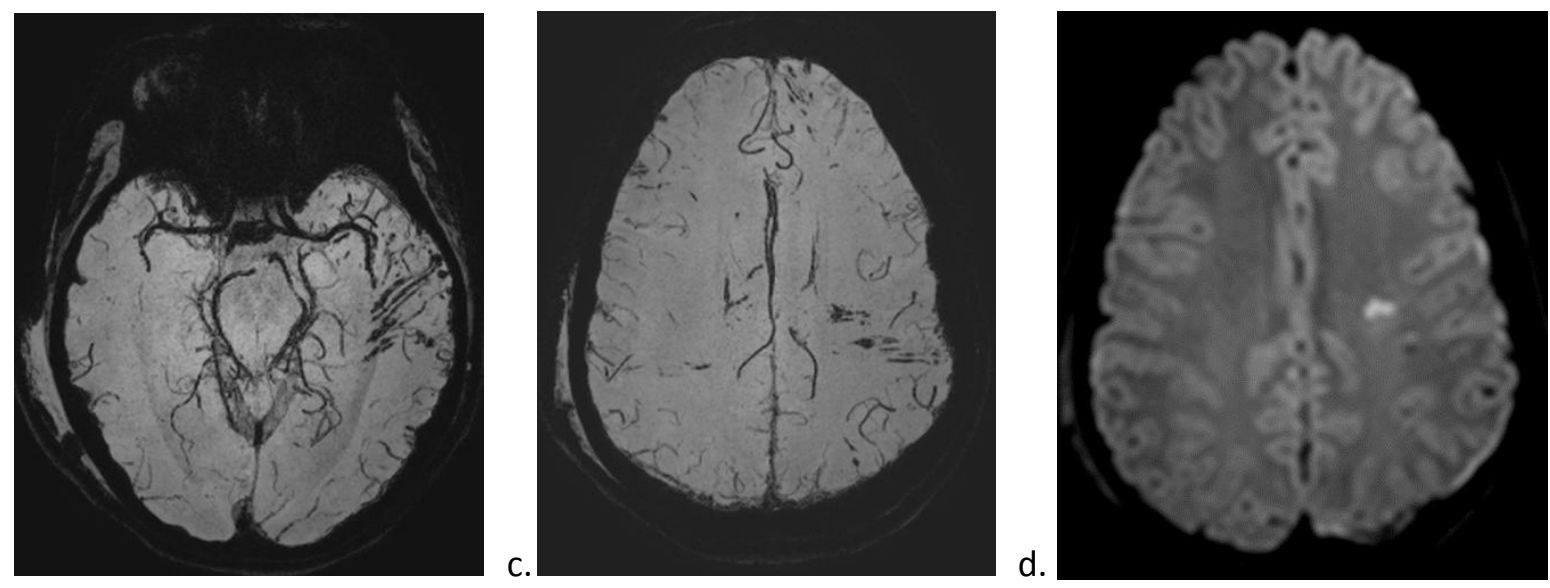
Figure 5. Scanning electron micrographs (SEM) of cortical vessels from a: control and b: TBI patient. There is flattening and reduction of the lumina of microvessels throughout, including the pial vessels, cortical arteries, and capillary zones. Changes are most pronounced in the superficial capillary zone. $1=$ =pial vessels; $2=$ long cortical artery; $3=$ middle cortical artery; $4=$ superficial capillary zone; $5=$ middle capillary zone; $6=$ deep capillary zone. Scale bar $=0.86 \mathrm{~mm}$ in (a) and $0.75 \mathrm{~mm}$ in (b).

FROM: Rodriguez-Baeza A, Reina-de la Torre F, Poca A et Al. Morphological features in human cortical brain microvessels after head injury: a three-dimiensional and immunocytochemical study. Anat Rec A Discov Mol Cell Evol Biol 2003; 273:583-593.
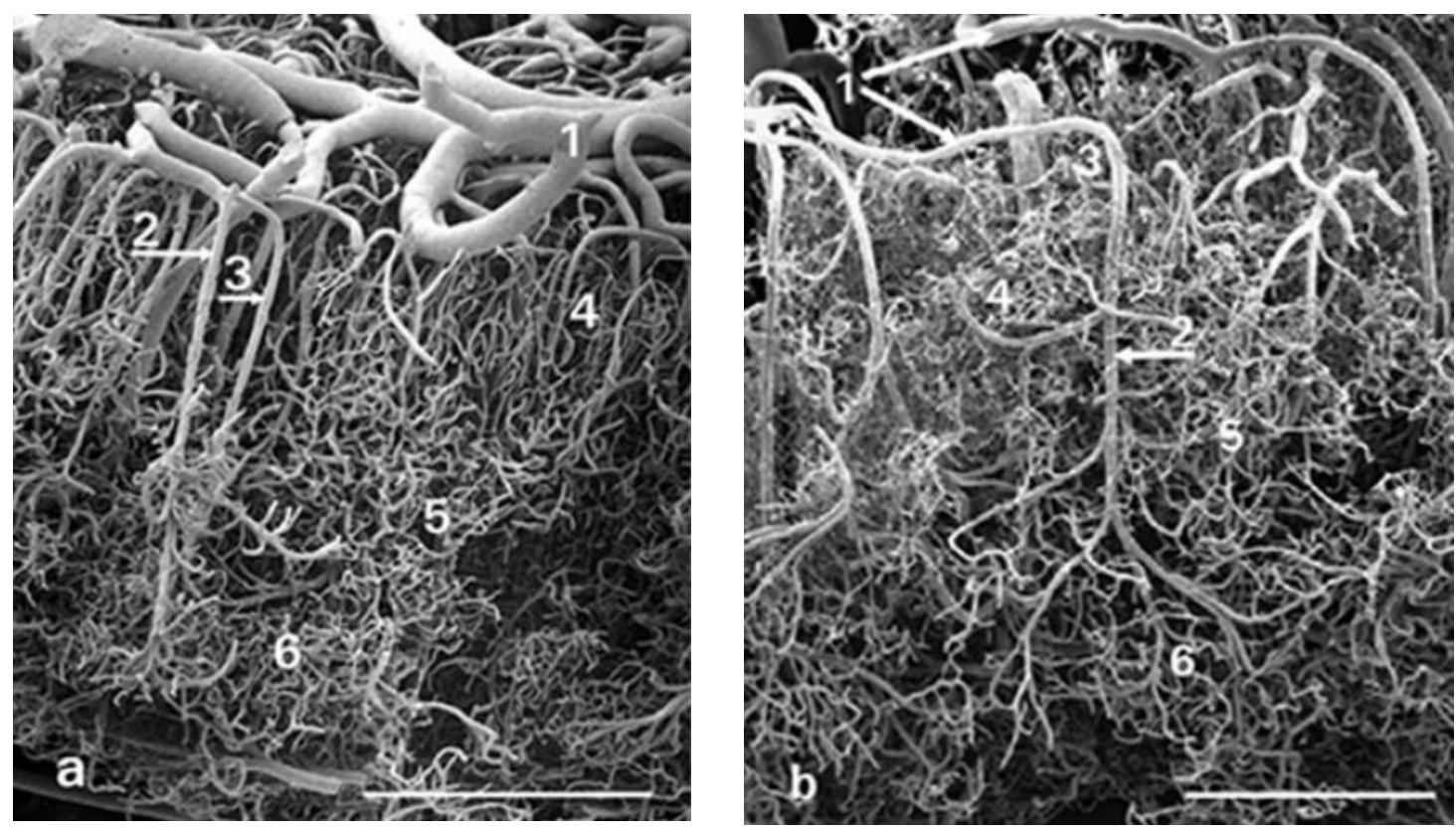
Figure 6. Scanning electron micrographs showing EM microvascular changes in 2 arteriolar vessels from the frontal lobe after TBI. a: arteriole with longitudinal folds (2') with transition from smooth to folds (arrowheads). 1=subpial zone; 2 =superficial zone; 3=cortical zone. Scale bar $=86 \mu \mathrm{m}$. b: arteriolar vessels (1) with longitudinal folds (2) and nuclear imprints of endothelial cells (3) at higher power. Scale bar $=23 \mu \mathrm{m}$.

Ref: Rodriguez-Baeza A, Reina-de la Torre F, Poca A et Al. Morphological features in human cortical brain microvessels after head injury: a three-dimiensional and immunocytochemical study. Anat Rec A Discov Mol Cell Evol Biol 2003; 273:583-593.
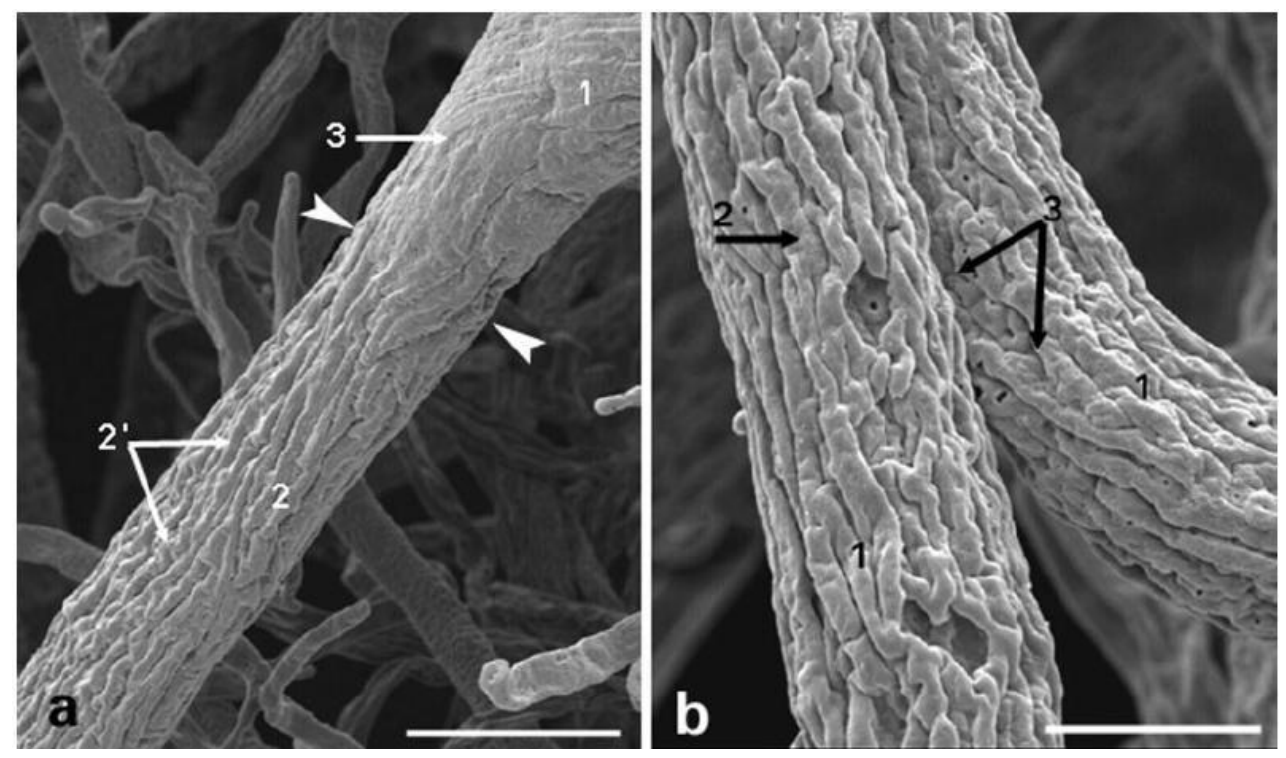
Figure 7. Blood vessel density at baseline, 6 hour and 14 days after experimental animal lateral fluid percussion injury (FPI) with immunohistochemical staining for rat endothelial cell antibody-1 (RECA-1) in representative perilesional cortical sections. A: control (no lesion), B: perilesional cortex 6 hours after injury, C: perilesional cortex 14 days after injury.

REF: Hayward NM, Tuunanen PI, Immonen R, et al. Magnetic resonance imaging of regional hemodynamic and cerebrovascular recovery after lateral fluid-percussion brain injury in rats. $\mathbf{J}$ Cereb Bl Flow \& Metab 2011; 31:166-177.
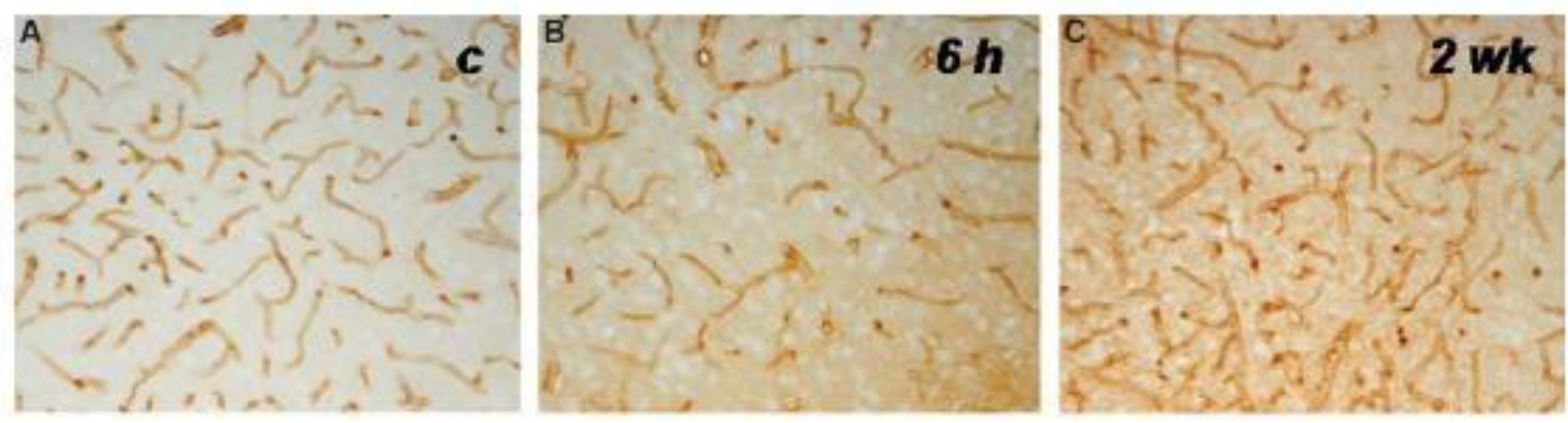
Figure 8. Top left: MRI-BOLD with 5\% $\mathrm{CO}_{2}$ hypercapnia challenge set-up with patient shown on the MRI stretcher and Douglas bag with $5 \% \mathrm{CO}_{2}$ at the side on the floor. Top right: Typical tracing of end tidal $\mathrm{CO}_{2}$ in blue and MRI-BOLD signal superimposed during MRI-BOLD with hypercapnia challenge. Bottom right: Typical CVR map generated with MRI-BOLD with hypercapnia challenge after coregistration with T1 MRI image.

REF: Courtesy of Franck Amyot, Ph.D., CNRM, USUHS

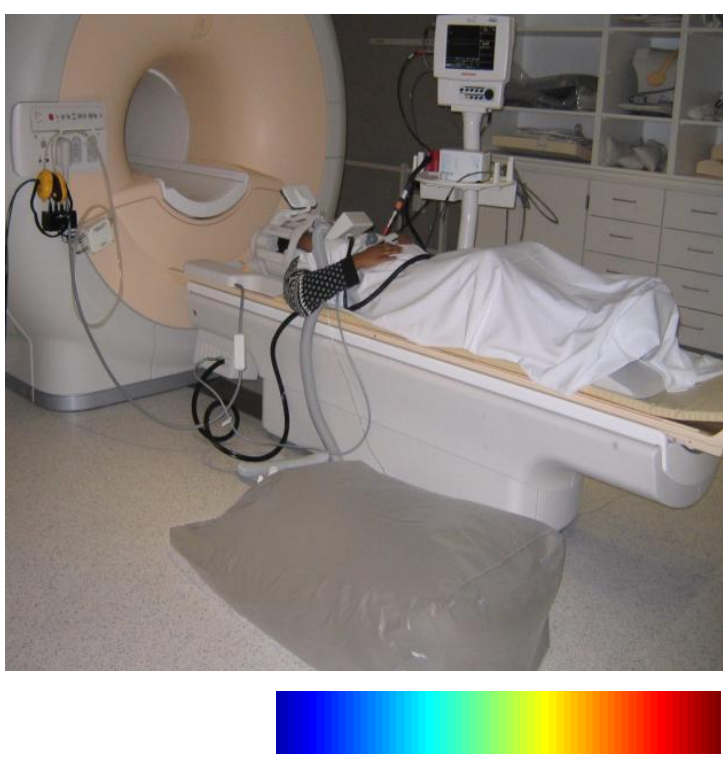

Detector
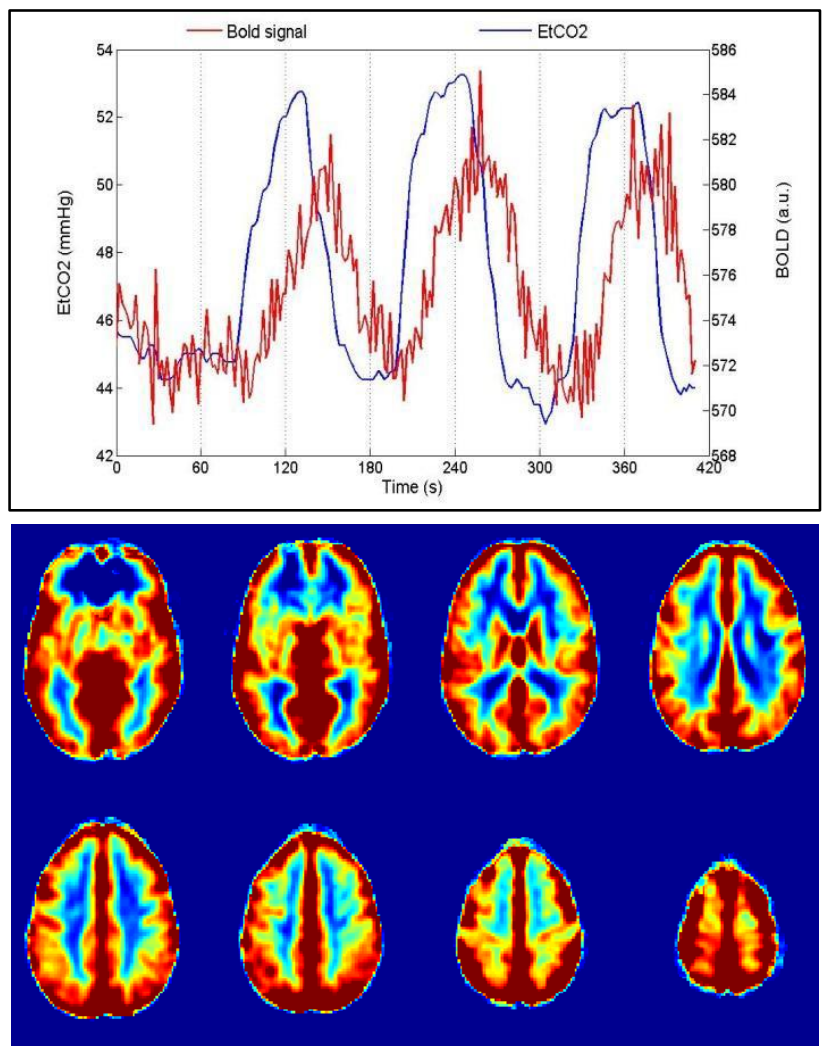
Figure 9. T1 MRI image (top) with corresponding cerebrovascular reactivity (CVR) map based on MRI-BOLD with 5\% hypercapnia challenge (bottom) in healthy control

REF: Courtesy of Franck Amyot, Ph.D., CNRM, USUHS (unpublished data)

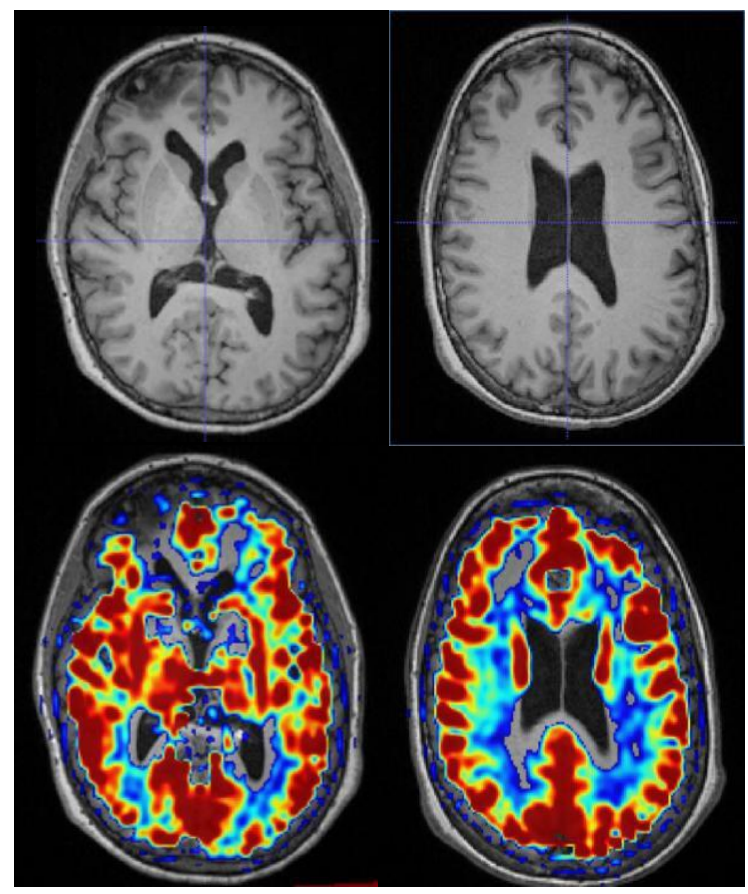


Figure 10. Cerebrovascular reactivity (CVR) histogram via MRI-BOLD with $5 \% \mathrm{CO}_{2}$ hypercapnia challenge in moderate and severe traumatic brain injury patients (red) compared to age-matched healthy controls (blue). The CVR histogram in TBI patients is both shifted to the left and has much greater variance. The CVR histogram in healthy controls is remarkably stable with very little intersubject variability

REF: Courtesy of Franck Amyot, Ph.D., CNRM, USUHS (unpublished data)

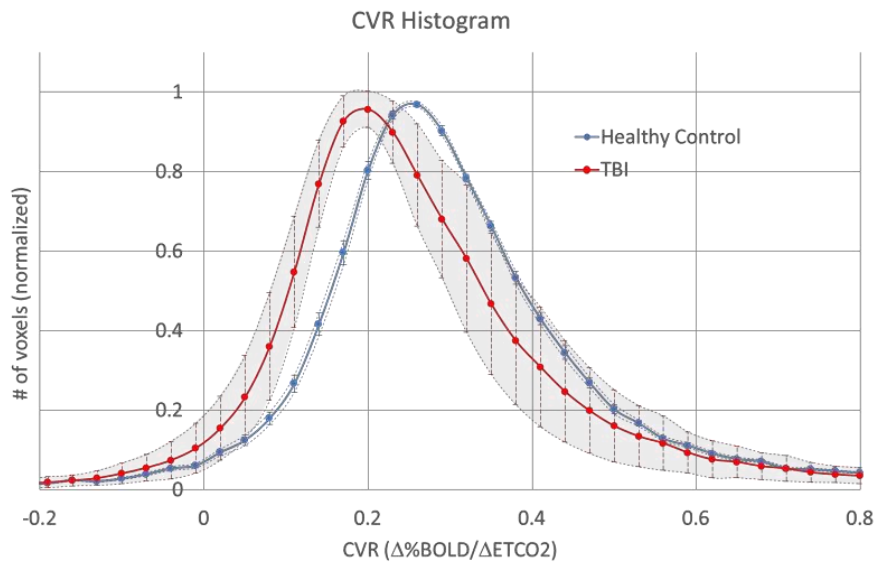


Figure 11. Correlation of CVR measurements via MRI-BOLD (with 5\% $\mathrm{CO}_{2}$ hypercapnia challenge via the Douglas Bag method) and fNIRS (Near InfraRed Spectroscopy with $5 \% \mathrm{CO}_{2}$ hypercapnia challenge via the Douglas Bag method) in traumatic brain injury (TBI) and healthy controls. The complementary methods give similar results with a high degree of correlation $\left(\mathrm{R}^{2}\right.$ $=0.7125)$ with lower and more variable CVR measurements in TBI patients compared to healthy controls.

REF: Courtesy of Franck Amyot, Ph.D., CNRM, USUHS (unpublished data)

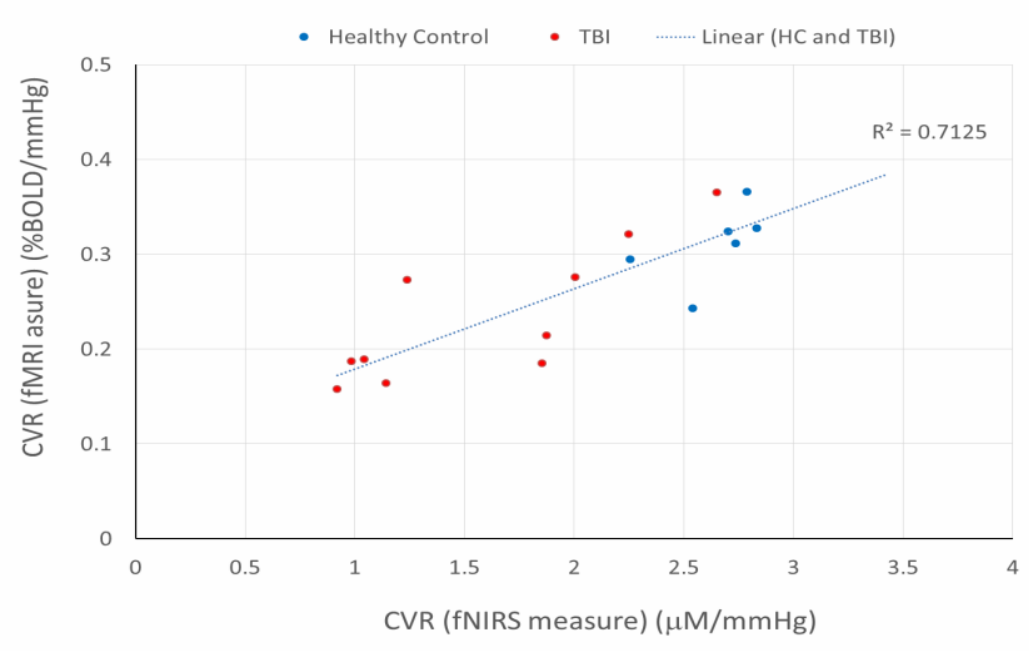

